Purdue University Purdue e-Pubs

$11-6-2015$

\title{
Stress and Energy Transmission by Inhomogeneous Plane Waves into Dissipative Media
}

Daniel C. Woods

Purdue University, woods41@purdue.edu

J Stuart Bolton

Purdue University, bolton@purdue.edu

Jeffrey F. Rhoads

Purdue University, jfrhoads@purdue.edu

Follow this and additional works at: http:// docs.lib.purdue.edu/herrick

Woods, Daniel C.; Bolton, J Stuart; and Rhoads, Jeffrey F., "Stress and Energy Transmission by Inhomogeneous Plane Waves into Dissipative Media" (2015). Publications of the Ray W. Herrick Laboratories. Paper 131.

http://docs.lib.purdue.edu/herrick/131

This document has been made available through Purdue e-Pubs, a service of the Purdue University Libraries. Please contact epubs@purdue.edu for additional information. 


\section{Stress and Energy Transmission by Inhomogeneous Plane Waves into Dissipative Media}

\section{Daniel C. Woods, J. Stuart Bolton, and Jeffrey F. Rhoads}

School of Mechanical Engineering,

Ray W. Herrick Laboratories, and Birck Nanotechnology Center,

Purdue University

West Lafayette, Indiana, USA

November 6, 2015

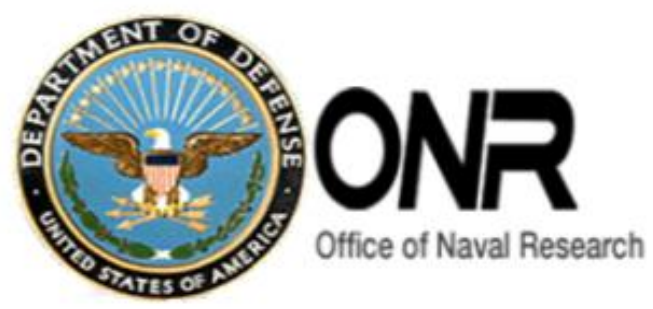




\section{Premise and Motivation}

- Detection of improvised explosive devices (IEDs)

- Strong dependence of vapor pressure on temperature

- May improve detection capabilities by selective heating

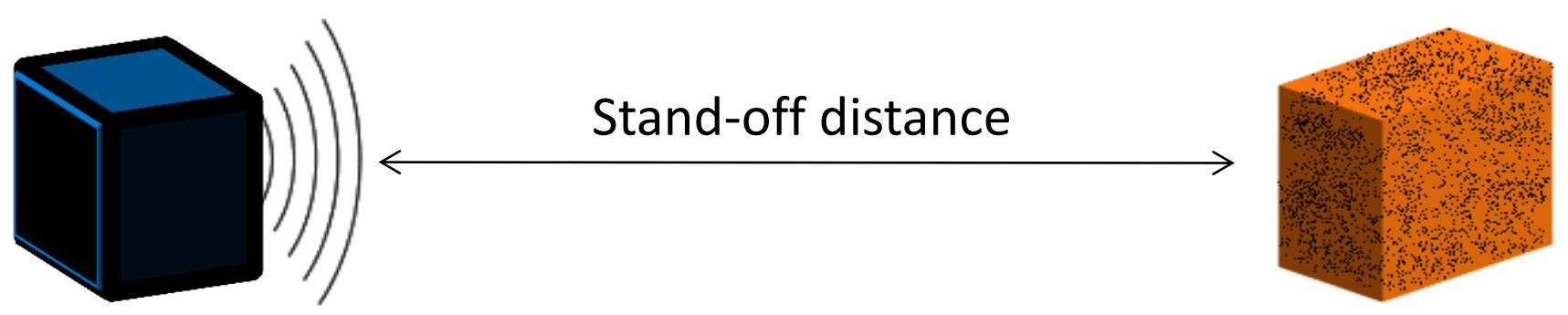

Low-frequency source
Energetic material

- Increase in temperature

- Increase in vapor pressure 


\section{Premise and Motivation}

- Optimization of incident wave parameters for maximal stress and energy transmission

- Theoretical study of incident inhomogeneous plane waves in dissipative media

- Minimization of reflection coefficient magnitude

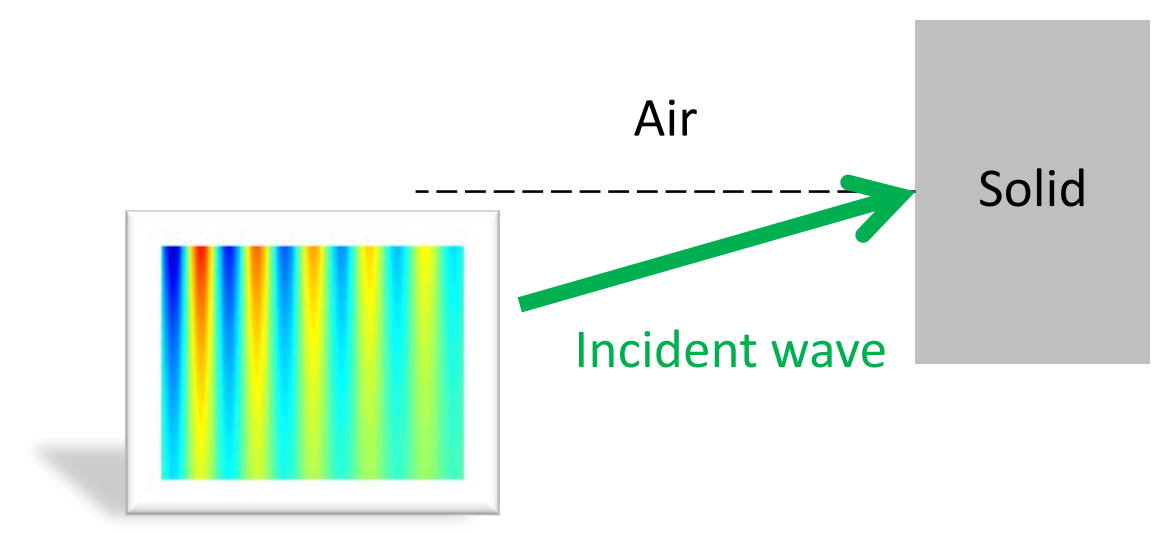

Basic representation of acoustical interface 


\section{General Acoustic Plane Waves}

Ideal fluid or Elastic solid

Homogeneous wave

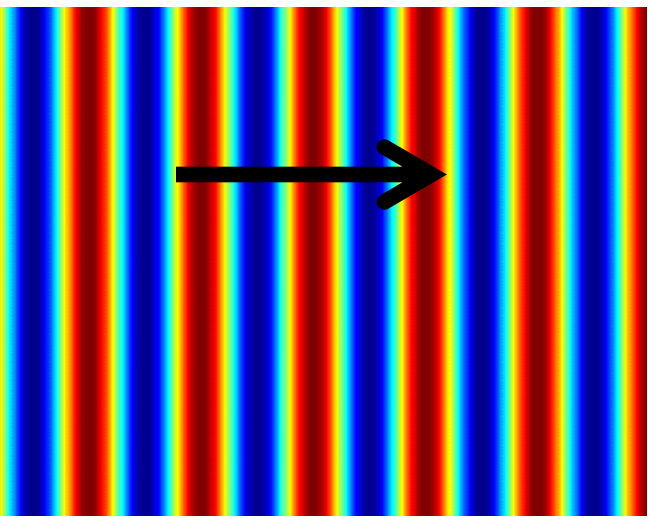

Inhomogeneous wave

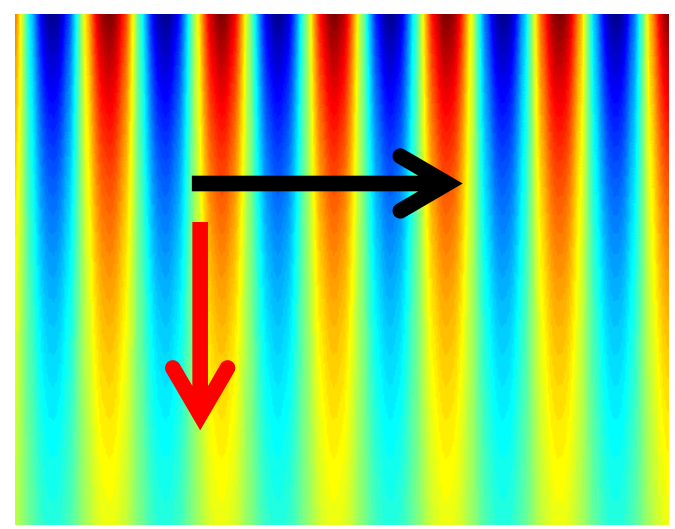

Real fluid or Viscoelastic solid
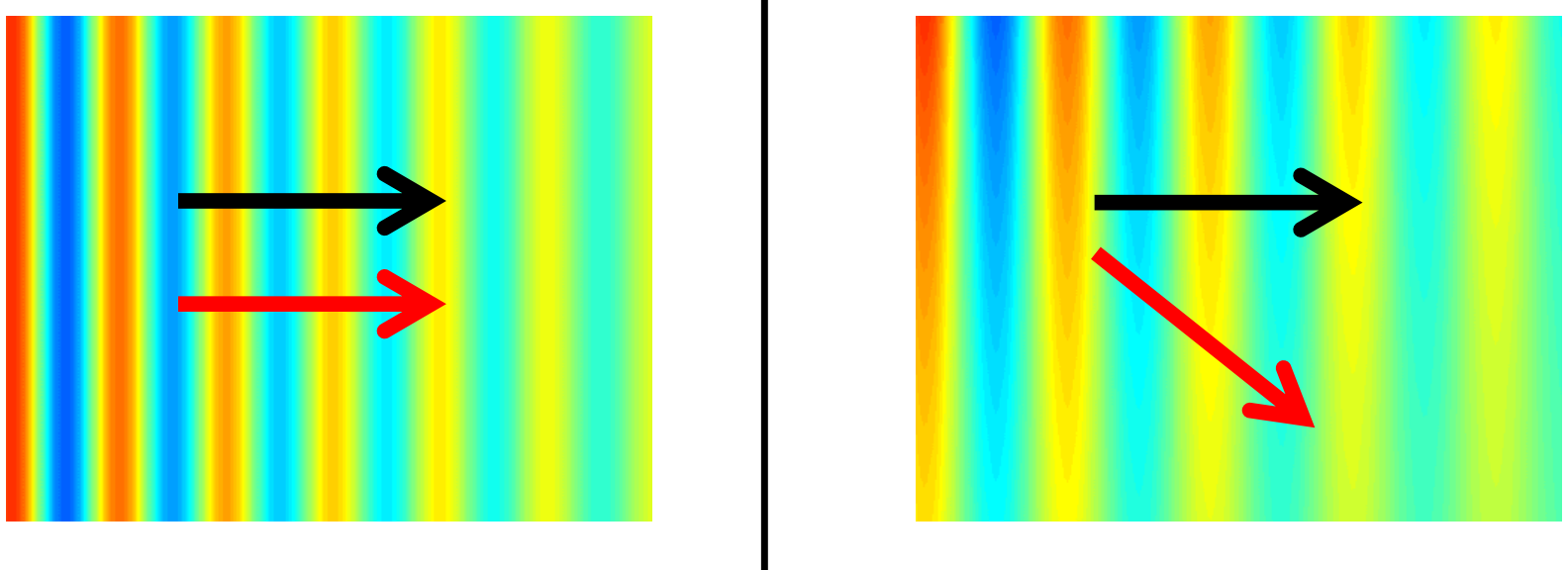


\section{Representation in Dissipative Media}
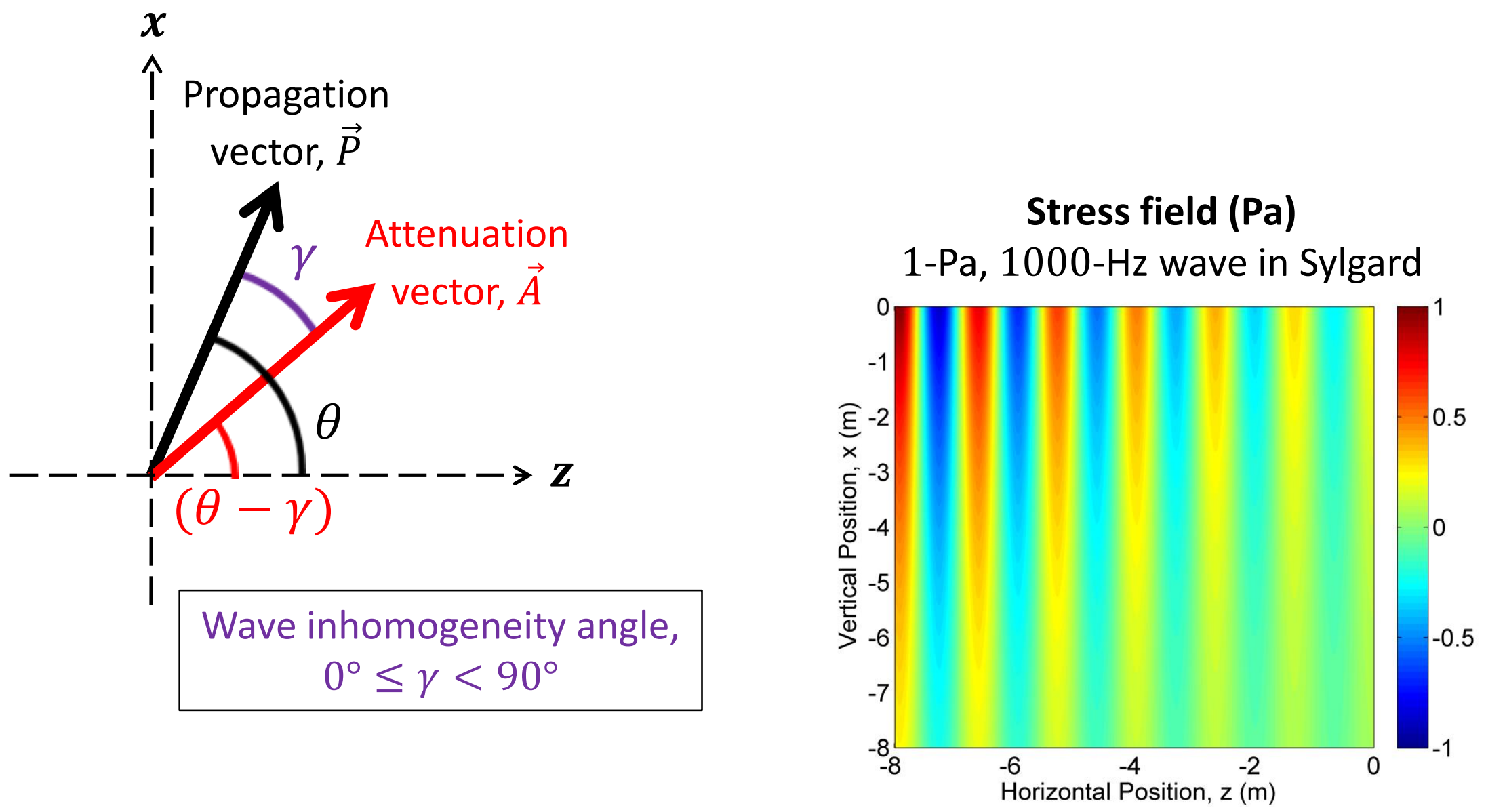


\section{Representation in Dissipative Media}

- Each wave type is characterized by the corresponding material wavenumber:

$$
\tilde{k}=k_{R}+j k_{I}
$$

- Homogeneous waves $\left(\gamma=0^{\circ}\right)$ :

$$
v_{H}=\frac{\omega}{k_{R}}, \quad\left|\vec{A}_{H}\right|=-k_{I}
$$

- Inhomogeneous waves $\left(\gamma \neq 0^{\circ}\right)$ :

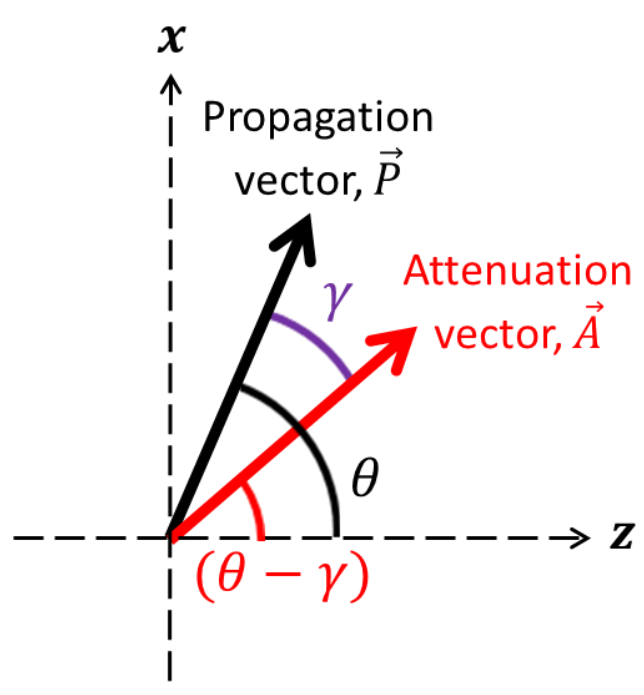

$$
\begin{gathered}
v=\text { function }(\gamma)<v_{H}, \\
|\vec{A}|=\operatorname{function}(\gamma)>\left|\vec{A}_{H}\right| \\
\tilde{k}_{x}=|\vec{P}| \sin (\theta)-j|\vec{A}| \sin (\theta-\gamma) \\
\tilde{k}_{z}=|\vec{P}| \cos (\theta)-j|\vec{A}| \cos (\theta-\gamma)
\end{gathered}
$$




\section{Creation of Inhomogeneous Plane Waves}
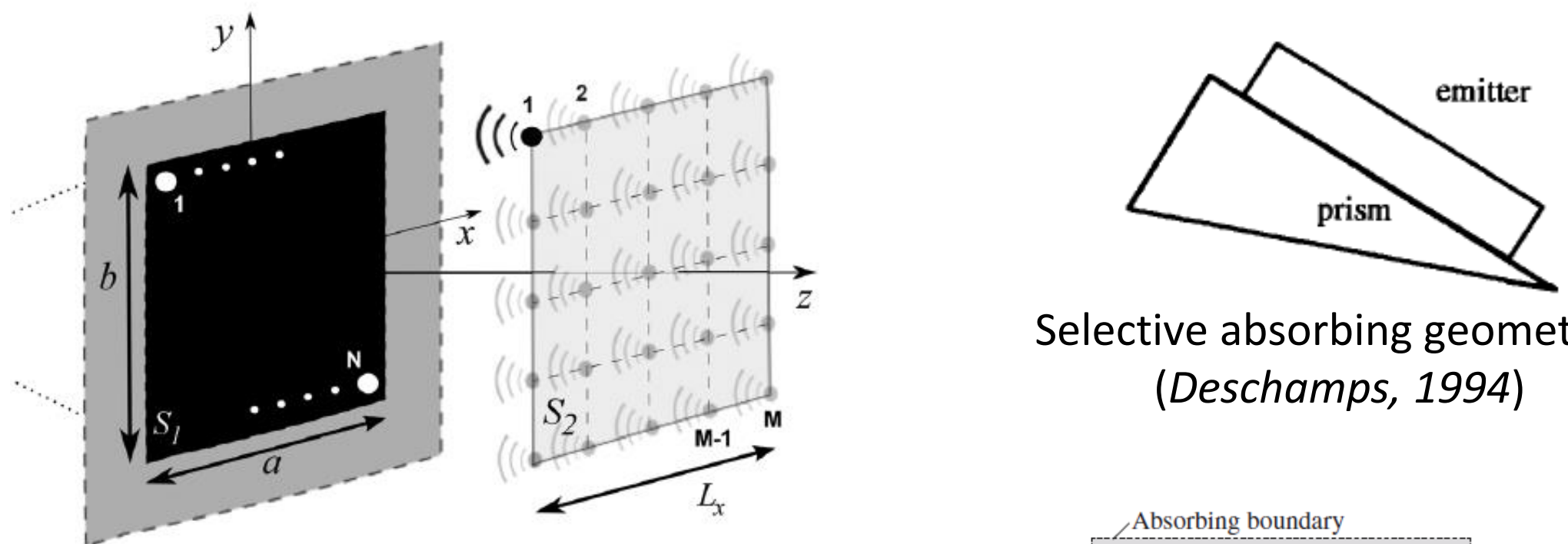

Selective absorbing geometries

(Deschamps, 1994)

Phased arrays of sources

(Robin et al., 2014)

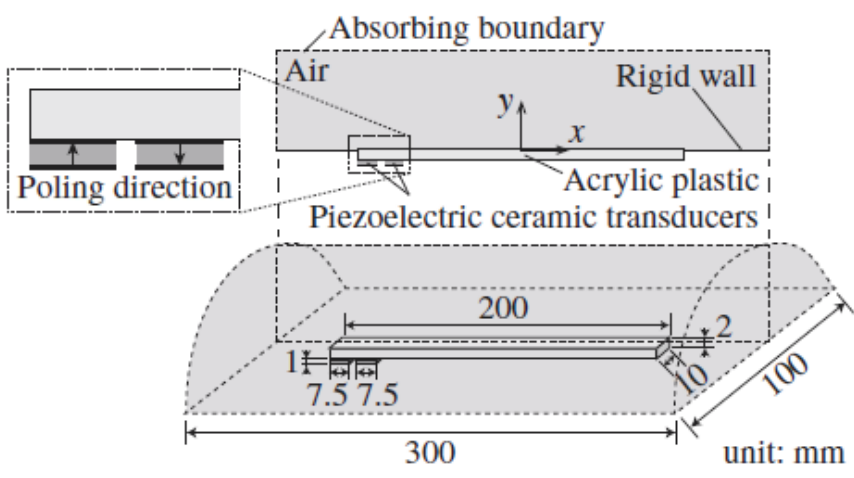

Vibrating plates coupled to piezoelectric transducers

(Fujii et al., 2014) 


\section{Fluid-Solid Interface}

- Boundary conditions at interface $(z=0)$

$$
\begin{aligned}
& \sigma_{1, z}(x, 0)=\sigma_{2, z}(x, 0) \\
& v_{1, z}(x, 0)=v_{2, z}(x, 0) \\
& \sigma_{2, x z}(x, 0)=0
\end{aligned}
$$

- Trace wavenumber continuity

$$
\tilde{k}_{1, x}=\tilde{k}_{2, x}=\tilde{\kappa}_{2, x}
$$

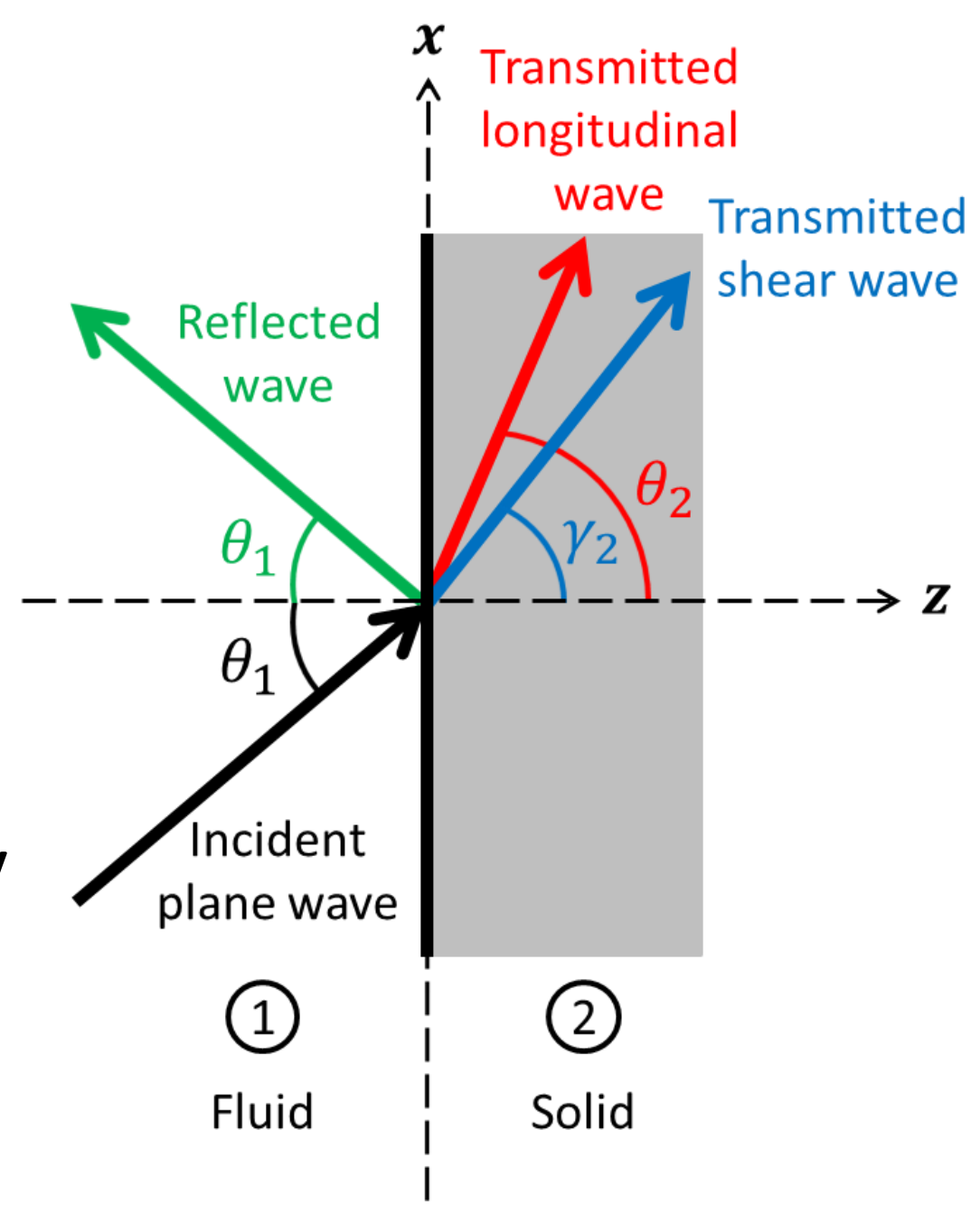




\section{Optimal Incident Wave Parameters}

- Optimal incidence angle is the Rayleigh angle

Wavefronts of incident wave
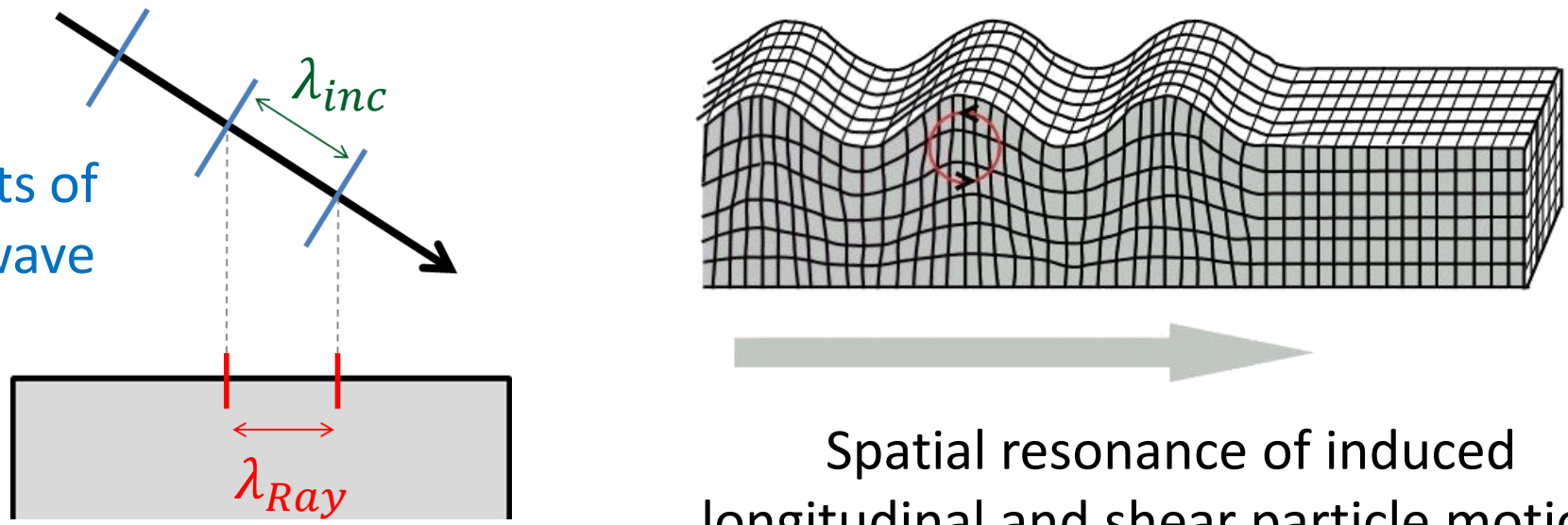

Spatial resonance of induced longitudinal and shear particle motions (image: http://www.sjvgeology.org/oil/Rayleigh_surface_waves2.gif)

- Wave inhomogeneity:

- For low-loss solids, optimal incident wave is inhomogeneous (unique such inhomogeneity)

- For higher-loss solids, optimal incident wave is homogeneous 


\section{Results for Ideal Fluid-Solid Interface}

Magnitude of Reflection Coefficient

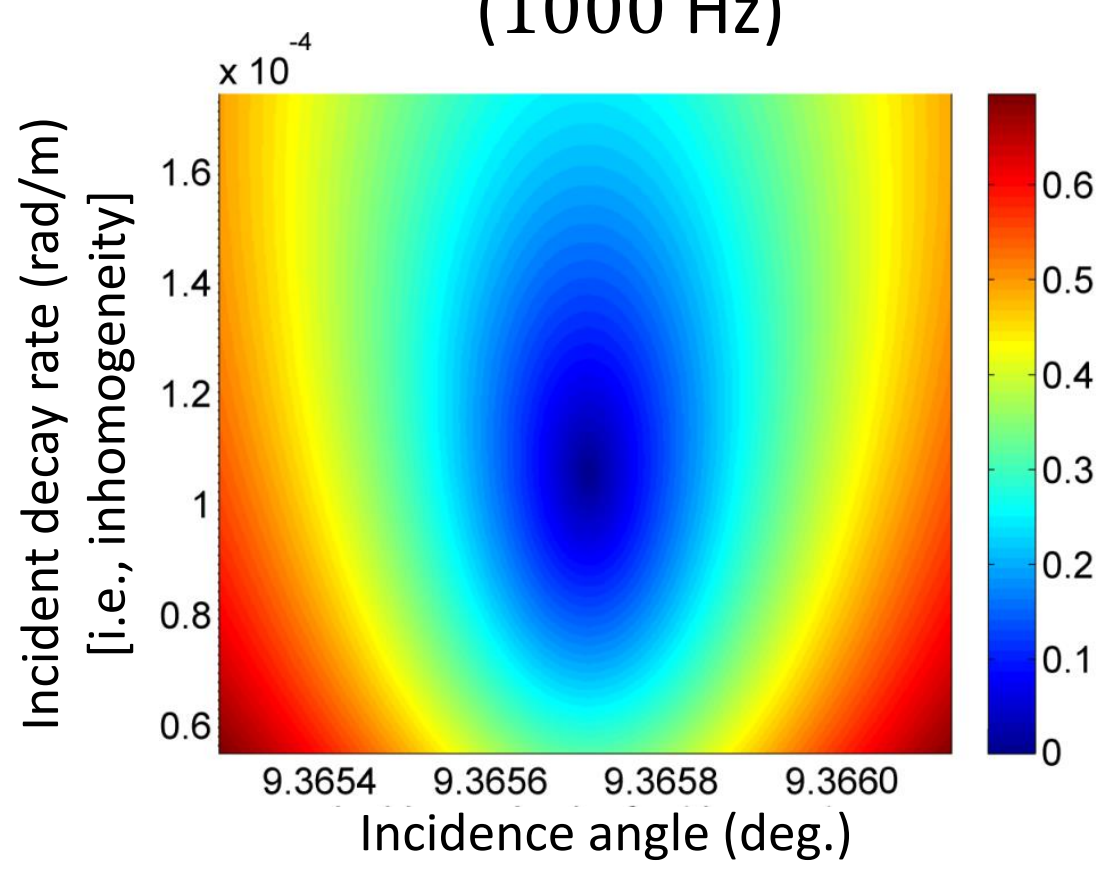

Stress Distribution in Elastic Solid (Pa)

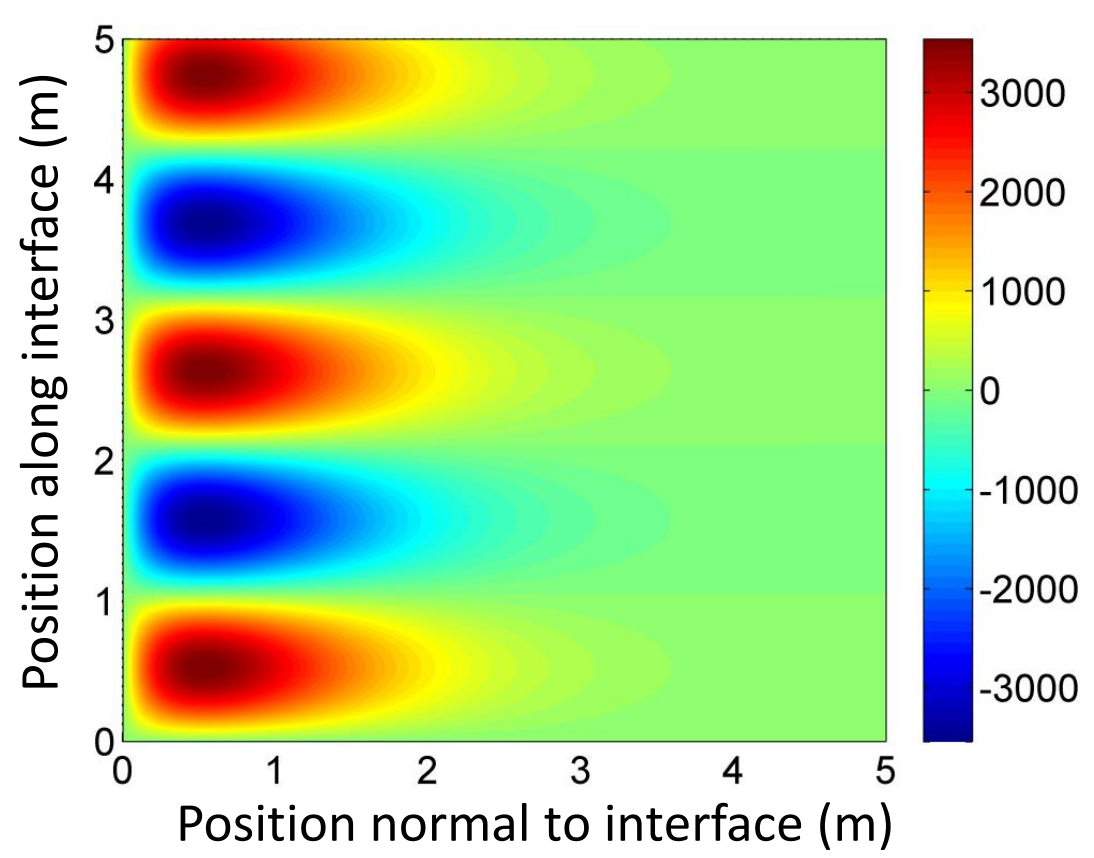




\section{Low-Loss Interface: Water-Stainless Steel}

\section{Magnitude of Reflection Coefficient}

Effect of Incidence Angle

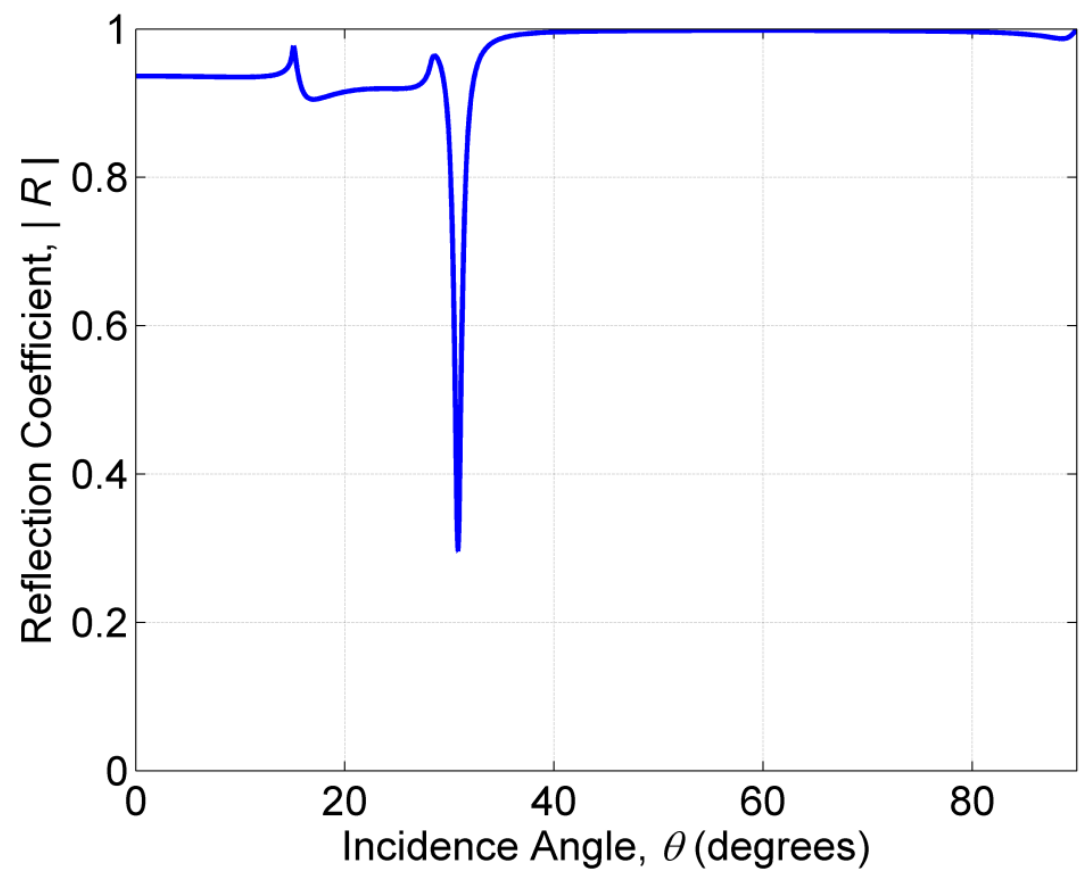

Effect of Wave Inhomogeneity (Near Rayleigh Angle)

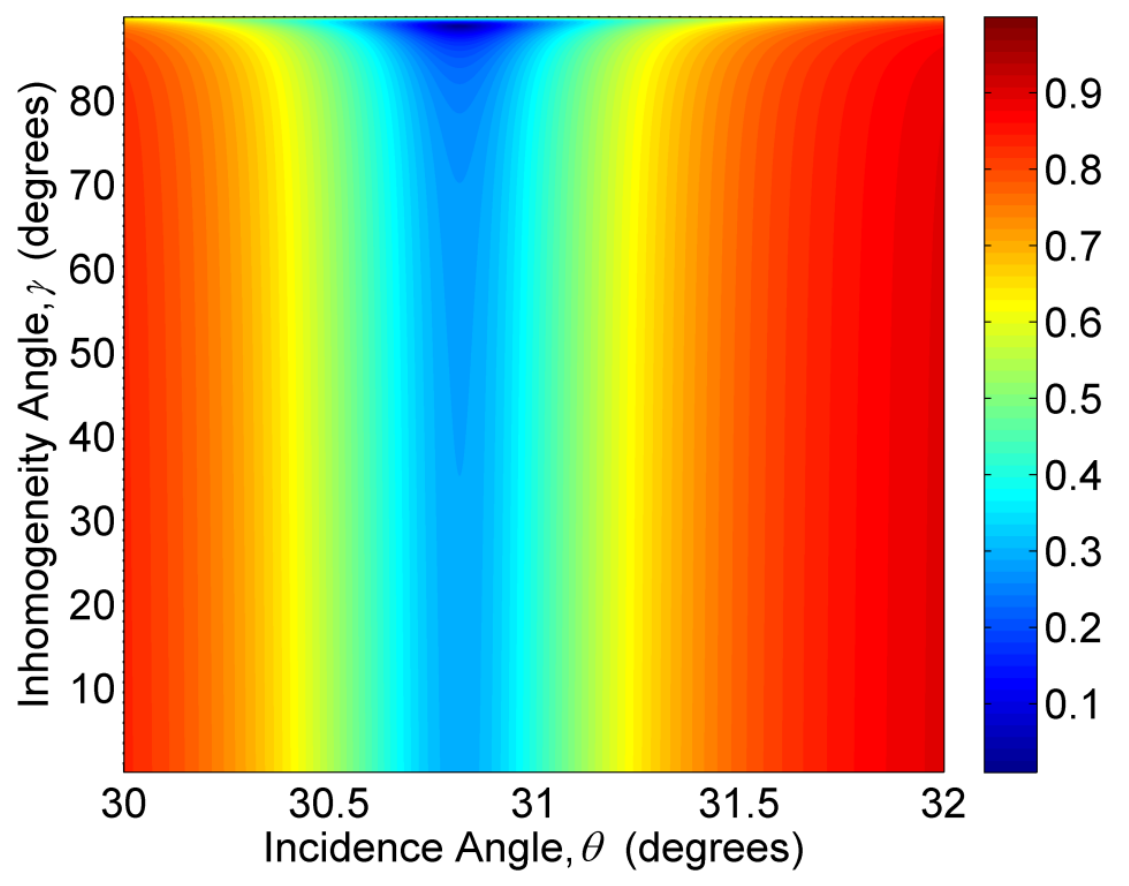




\section{Low-Loss Interface: Water-Stainless Steel}

\section{Magnitude of Reflection Coefficient}

Effect of Incidence Angle

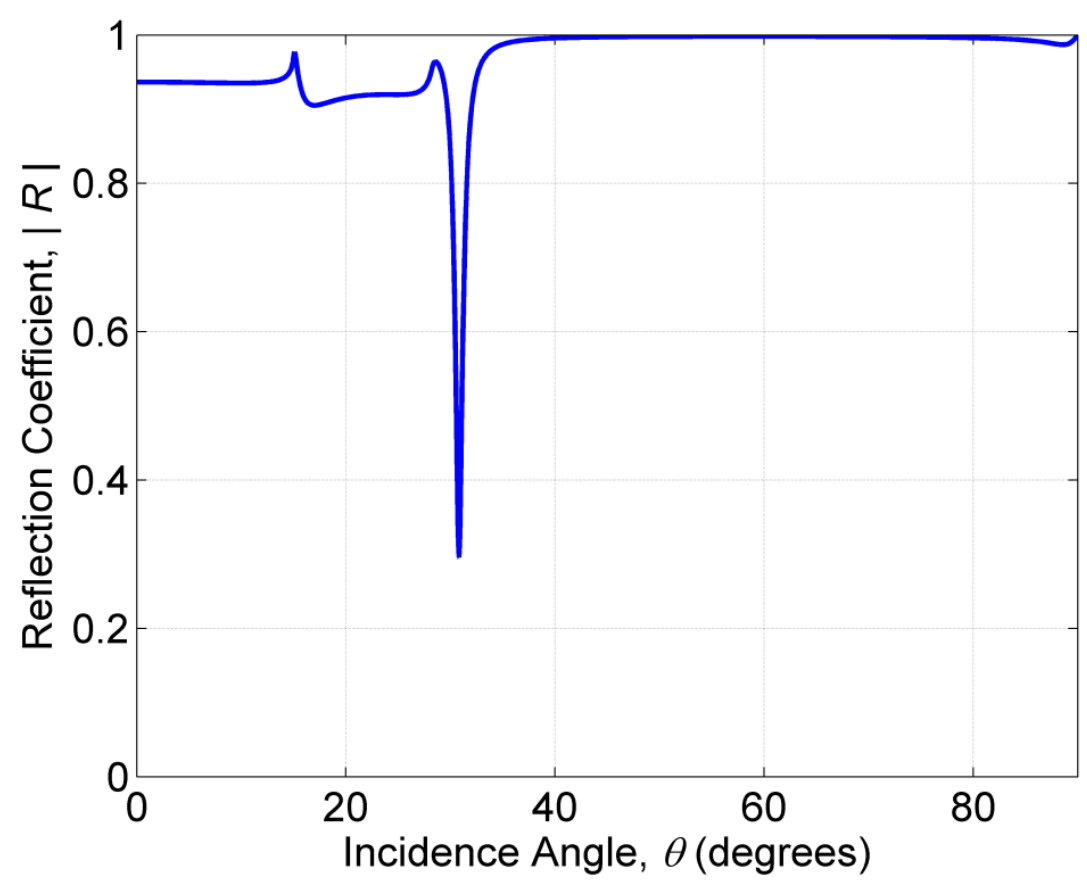

Effect of Wave Inhomogeneity (Near Rayleigh Angle)

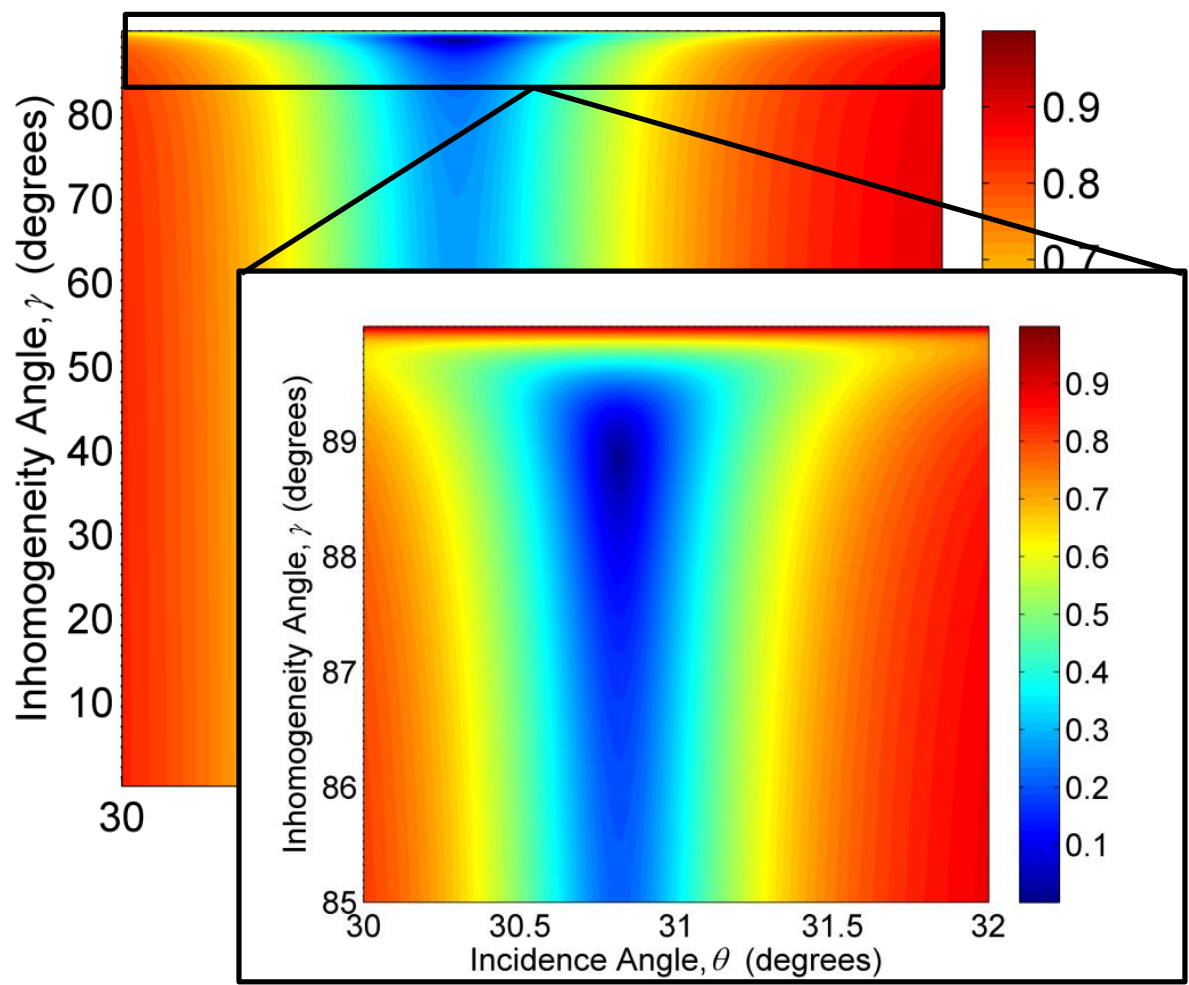




\section{Effect of Increasing Material Dissipation}

Magnitude of Reflection Coefficient vs. Dissipation Level $\left(\theta \approx 30.82^{\circ}\right)$
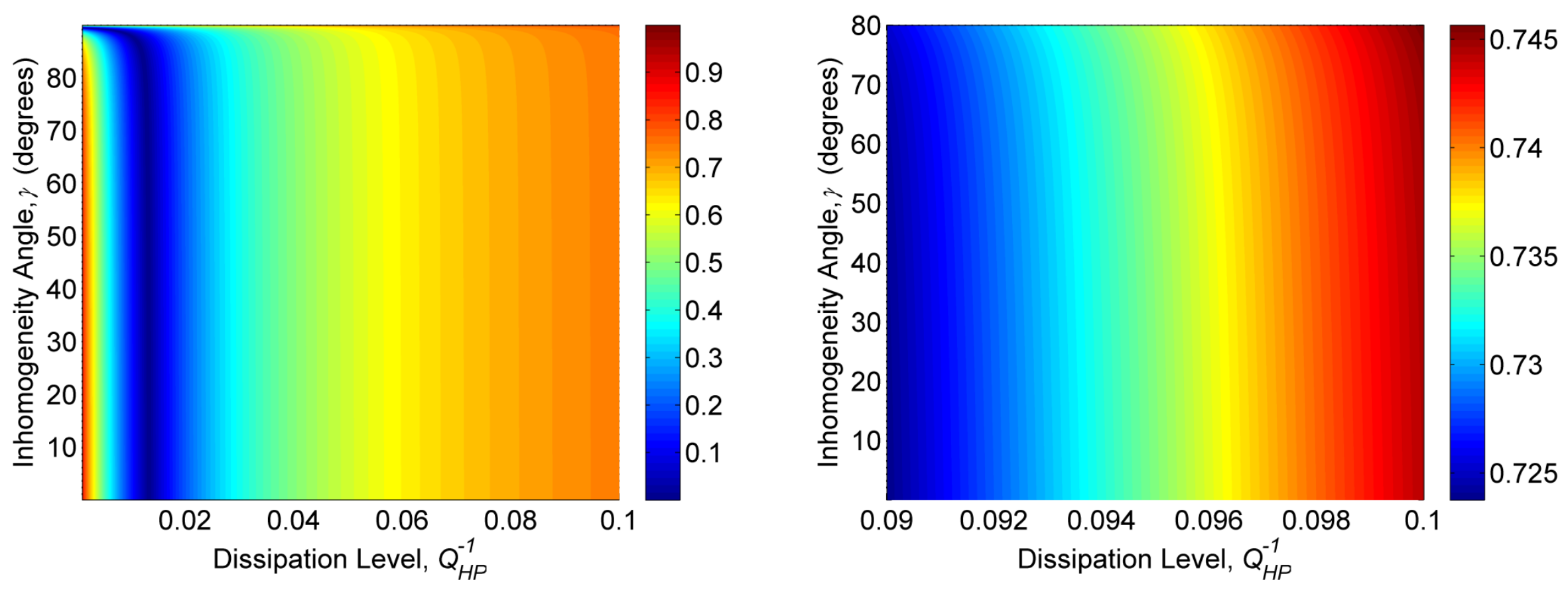


\section{Low-Loss Interface: Water-Stainless Steel}

$|\tilde{R}|$

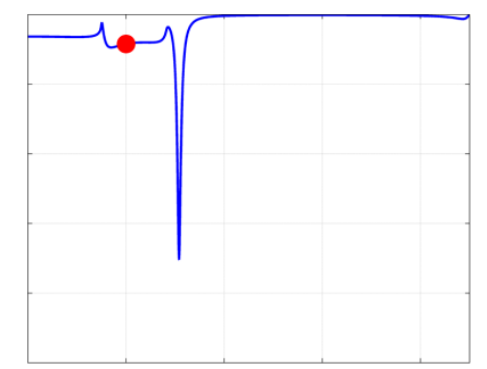

Incidence Angle, $\theta$

\section{Transmitted Normal Stress Distribution}

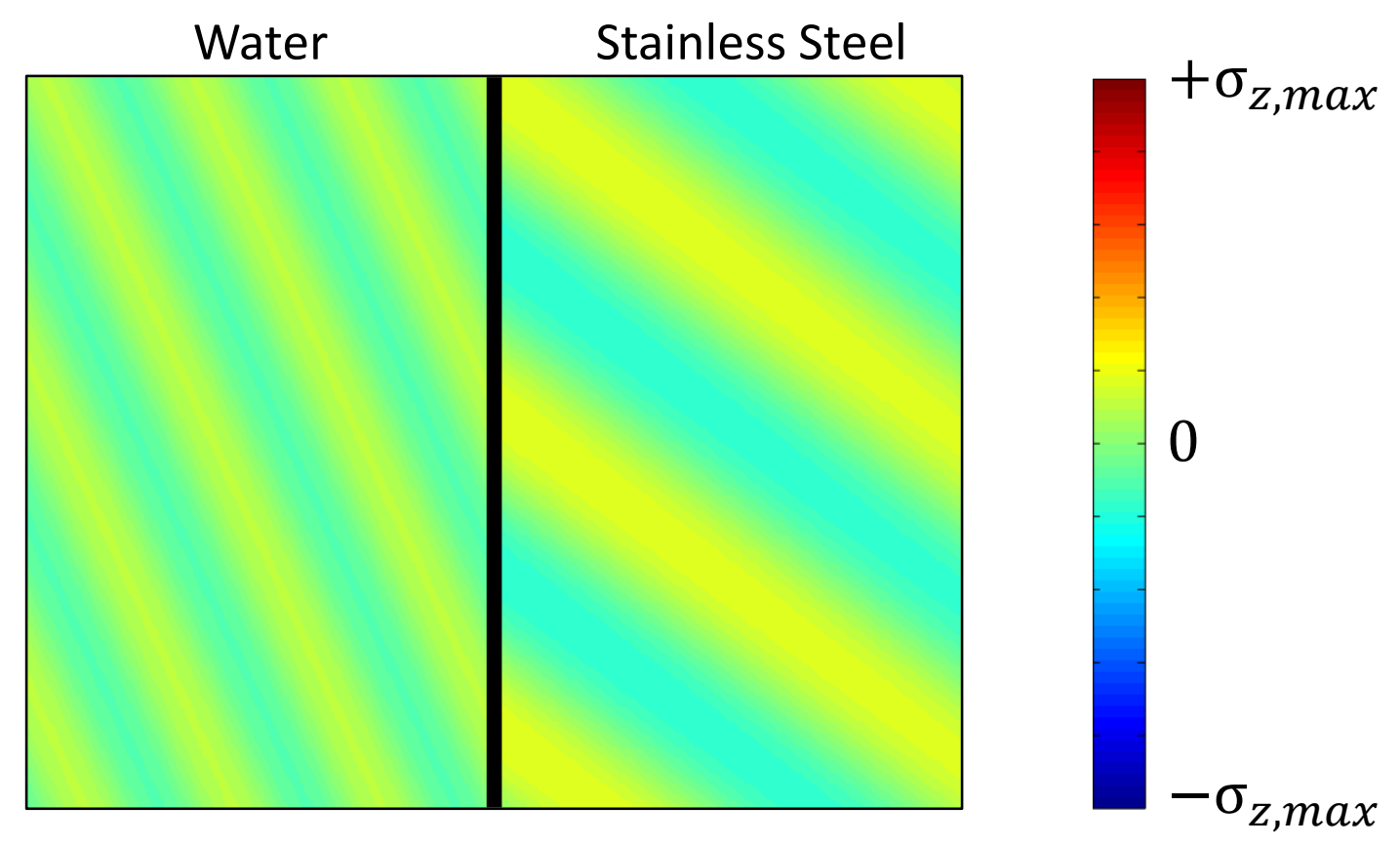




\section{Low-Loss Interface: Water-Stainless Steel}

$|\tilde{R}|$

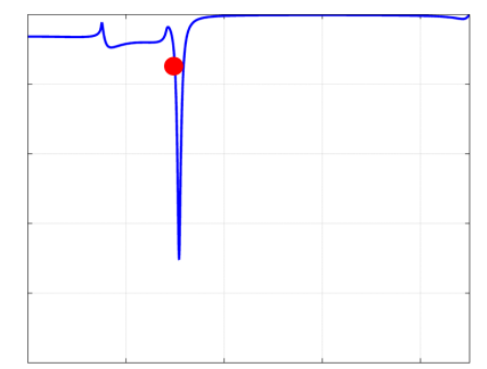

Incidence Angle, $\theta$

\section{Transmitted Normal Stress Distribution}
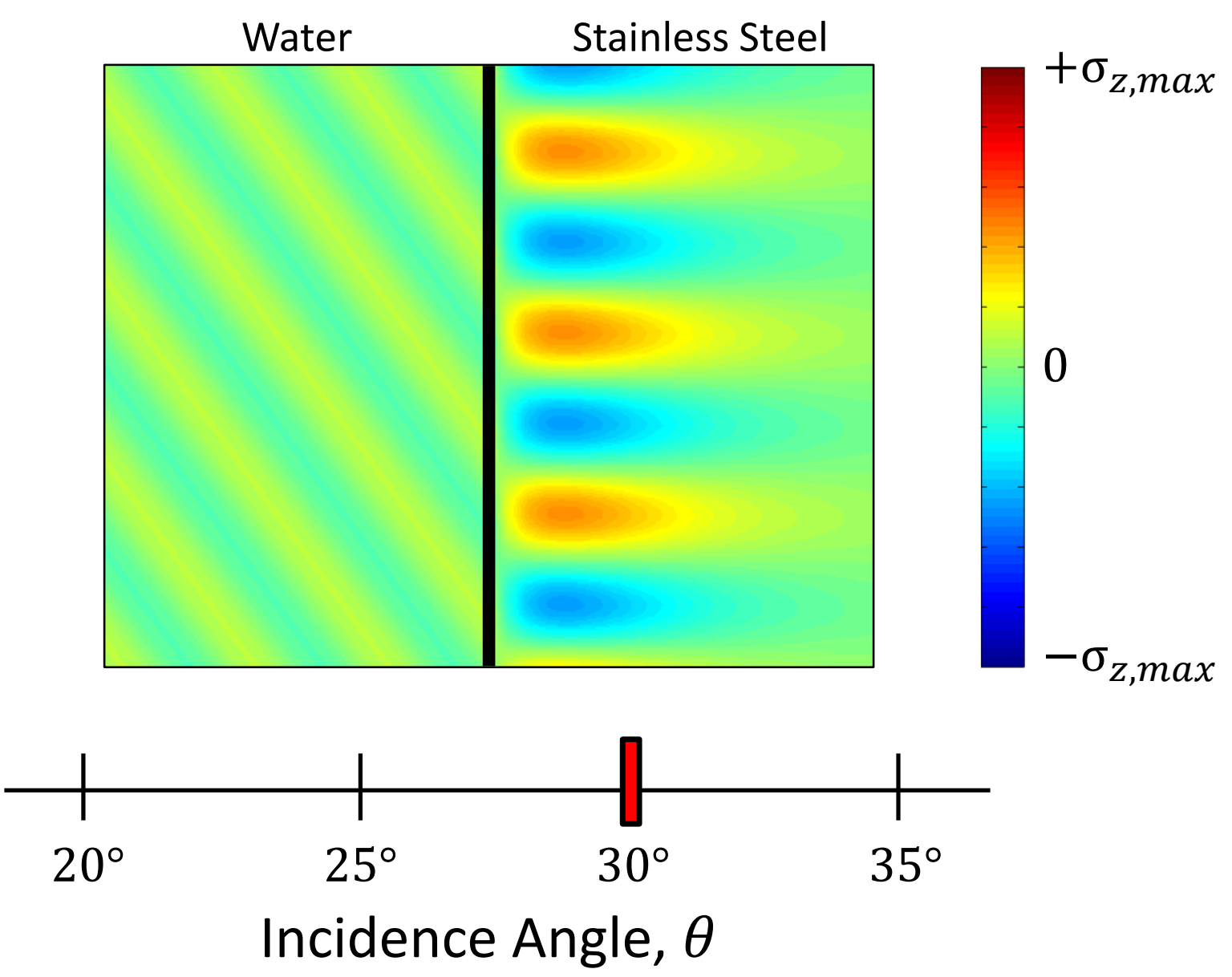


\section{Low-Loss Interface: Water-Stainless Steel}

$|\tilde{R}|$

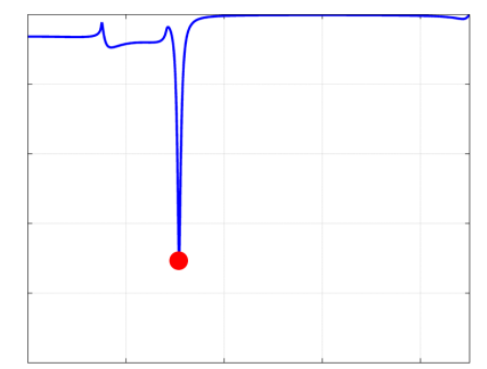

Incidence Angle, $\theta$

\section{Transmitted Normal Stress Distribution}

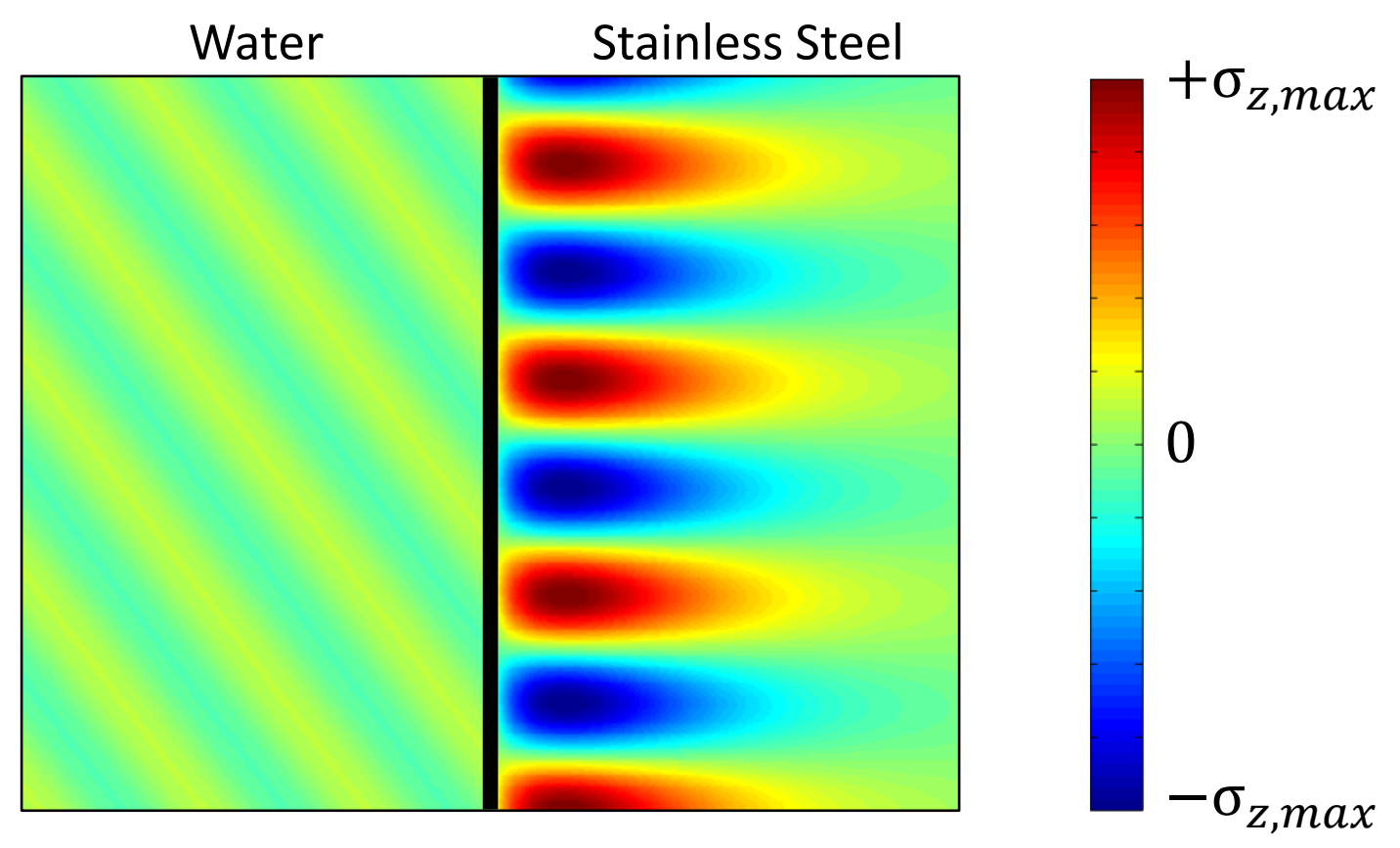




\section{Low-Loss Interface: Water-Stainless Steel}

$|\tilde{R}|$

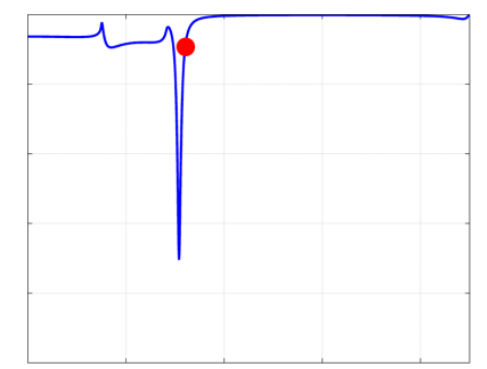

Incidence Angle, $\theta$

\section{Transmitted Normal Stress Distribution}

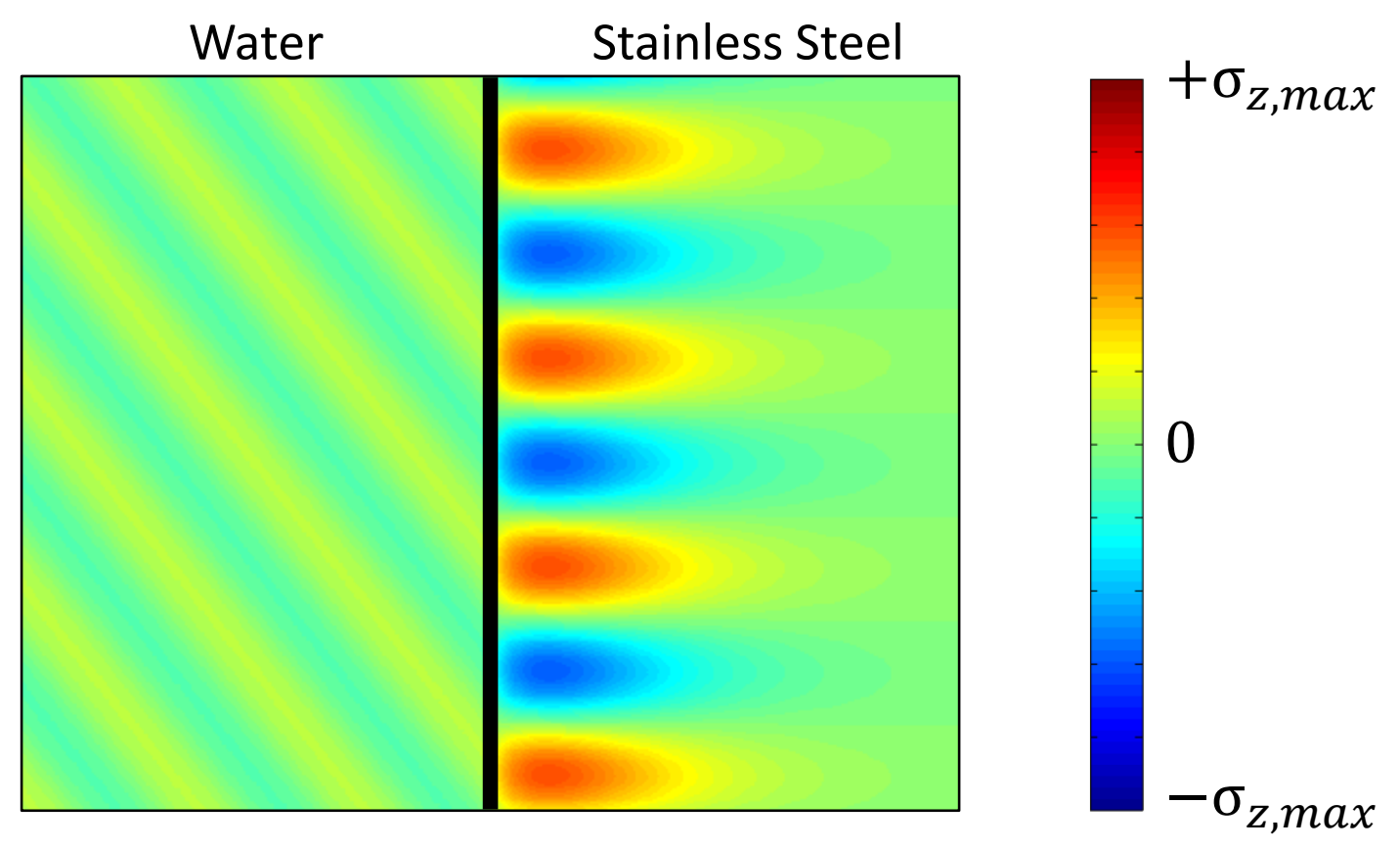




\section{Low-Loss Interface: Water-Stainless Steel}

$|\tilde{R}|$

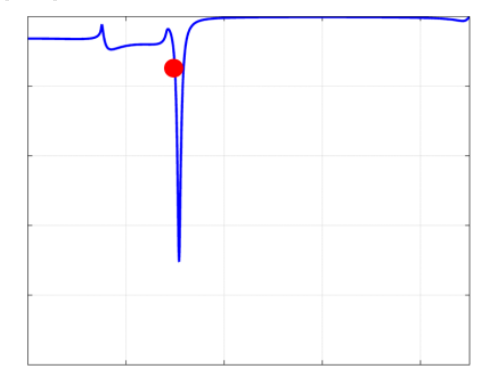

Incidence Angle, $\theta$

\section{Transmitted Normal Intensity Distribution}
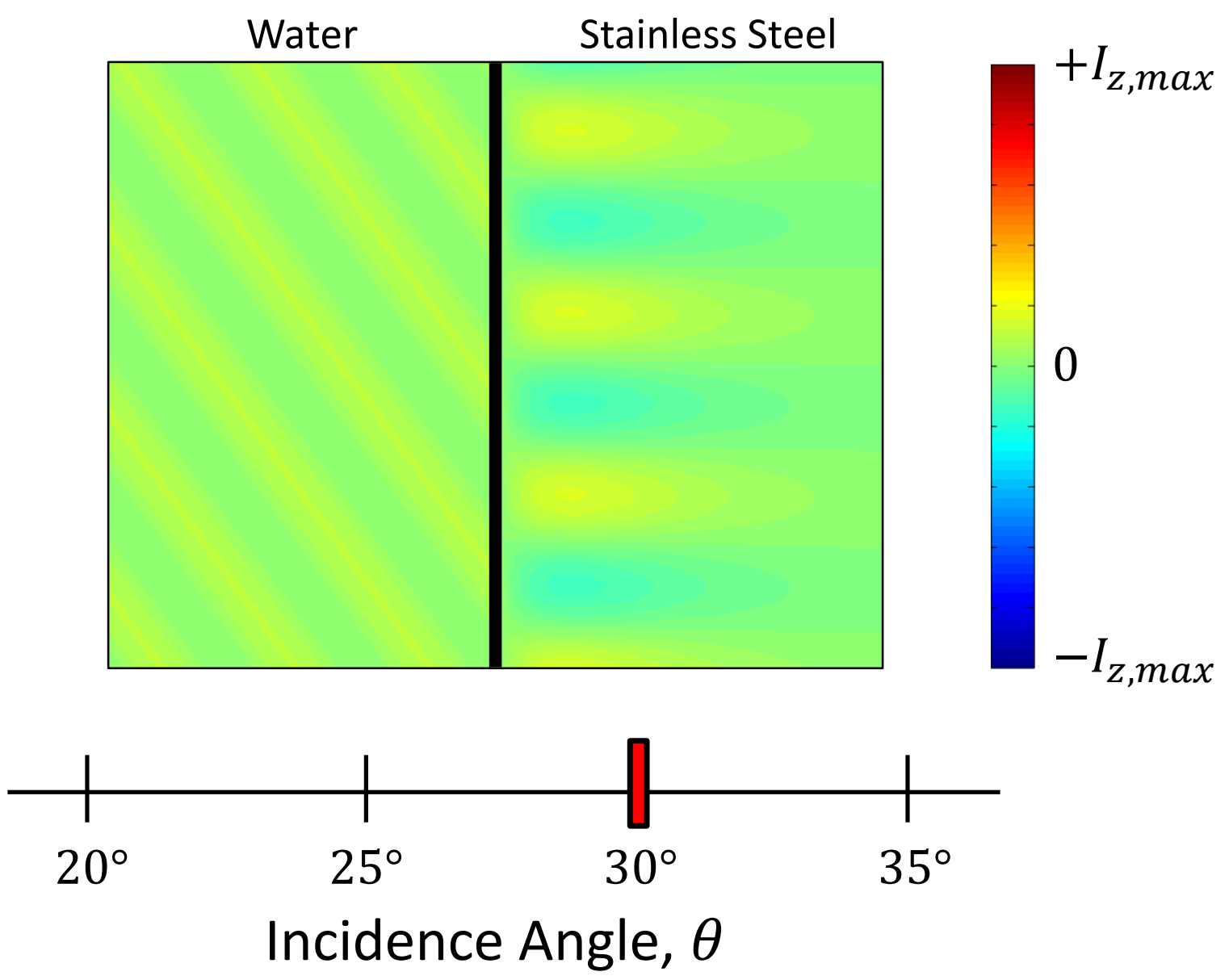


\section{Low-Loss Interface: Water-Stainless Steel}

$|\tilde{R}|$

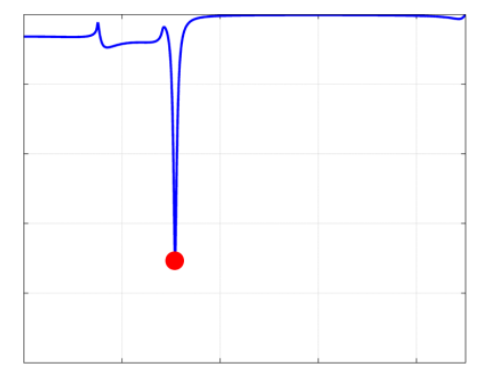

Incidence Angle, $\theta$

\section{Transmitted Normal Intensity Distribution}
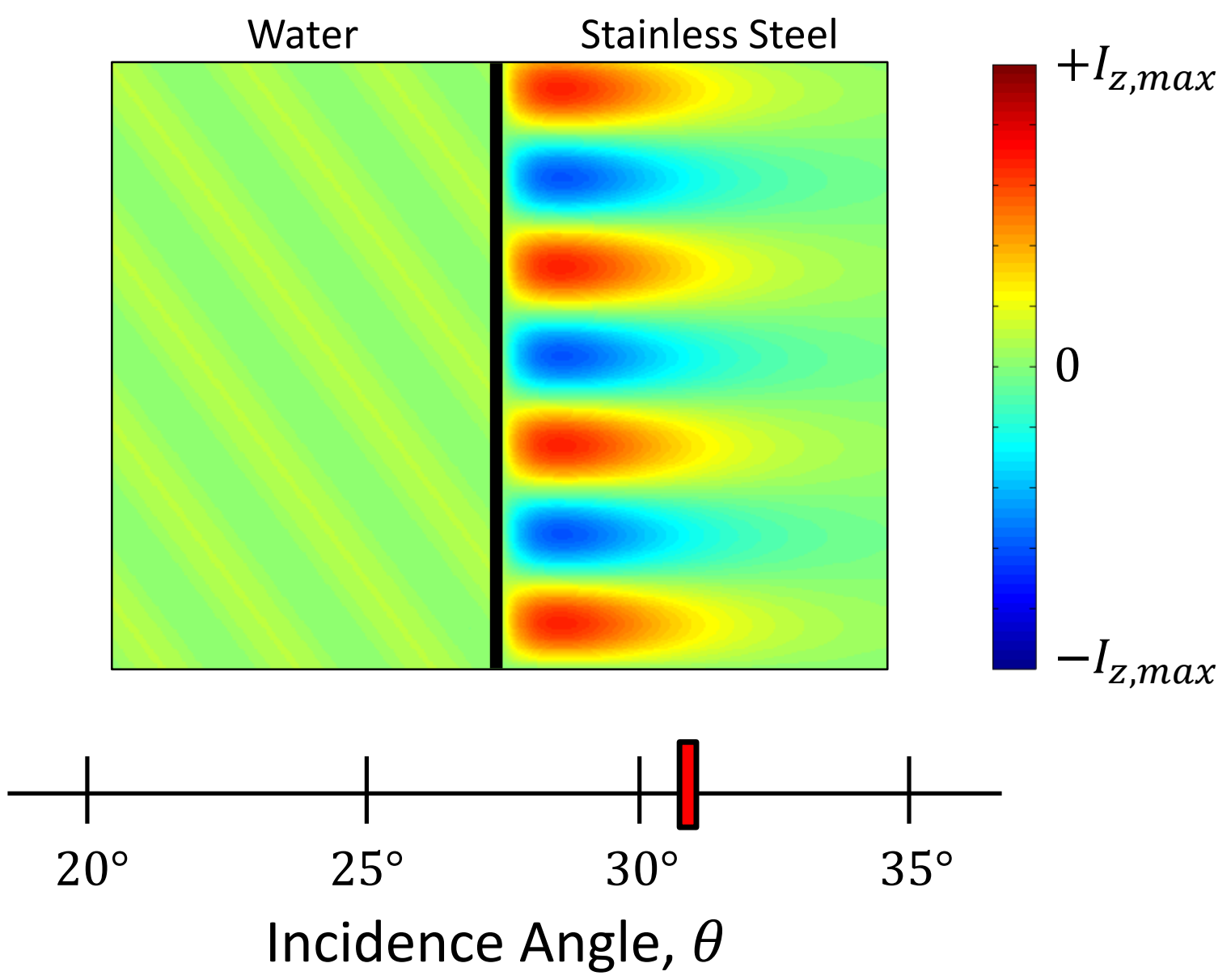


\section{Low-Loss Interface: Water-Stainless Steel}

$|\tilde{R}|$

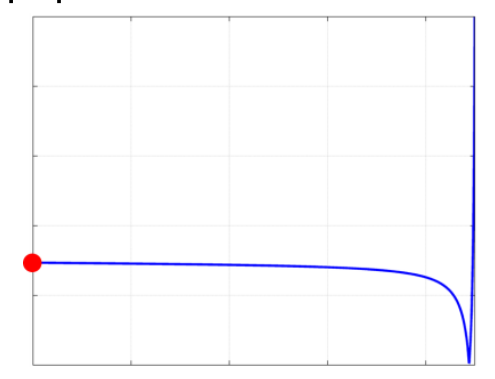

Inhomogeneity Angle, $\gamma$

\section{Transmitted Normal Intensity Distribution}
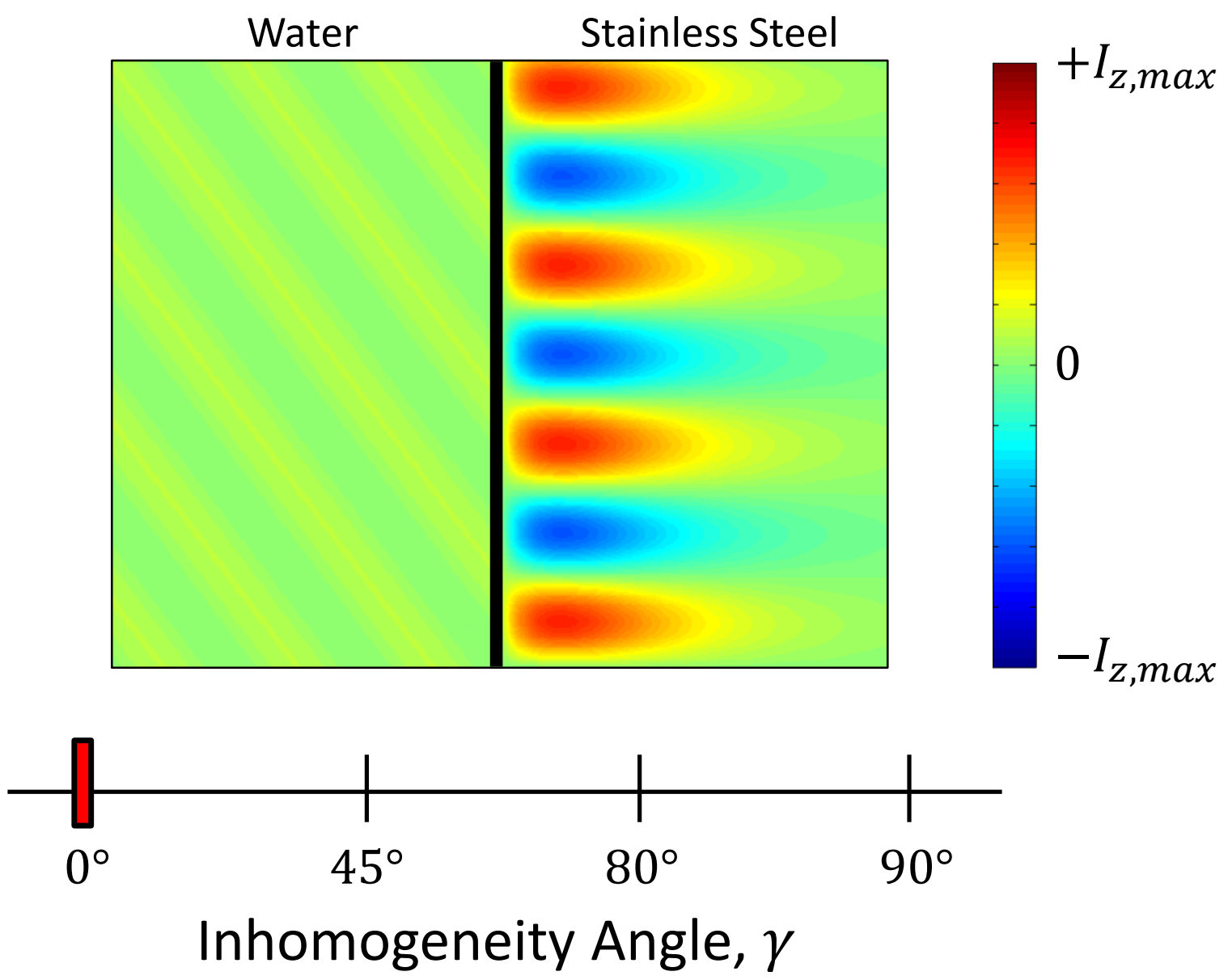


\section{Low-Loss Interface: Water-Stainless Steel}

$|\tilde{R}|$

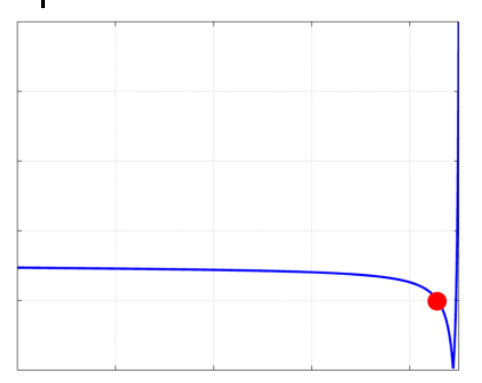

Inhomogeneity Angle, $\gamma$

\section{Transmitted Normal Intensity Distribution}
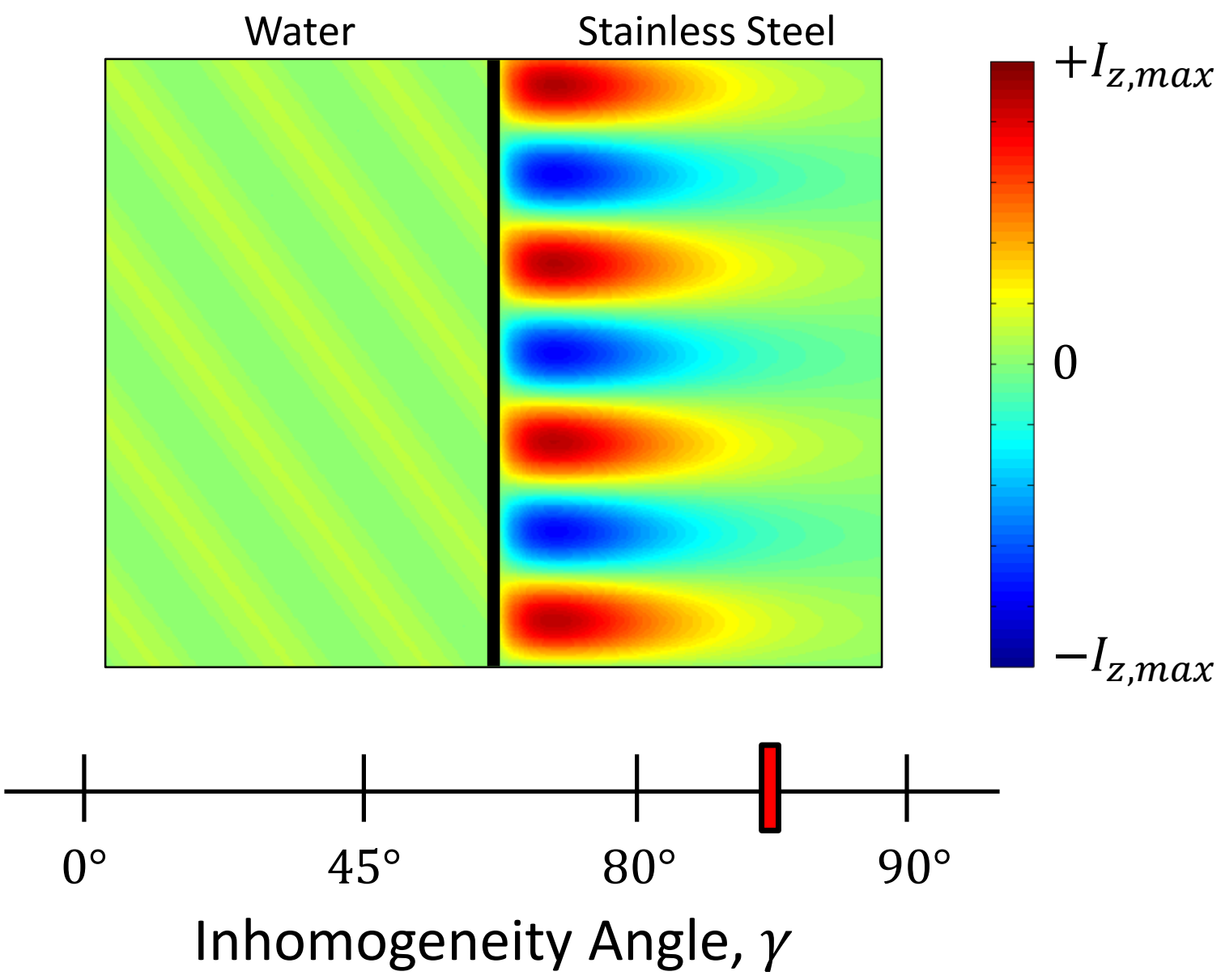


\section{Low-Loss Interface: Water-Stainless Steel}

$|\tilde{R}|$

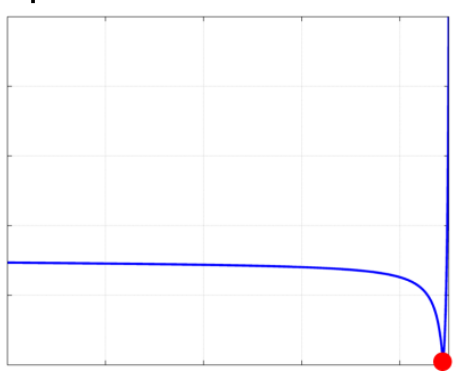

Inhomogeneity Angle, $\gamma$

\section{Transmitted Normal Intensity Distribution}
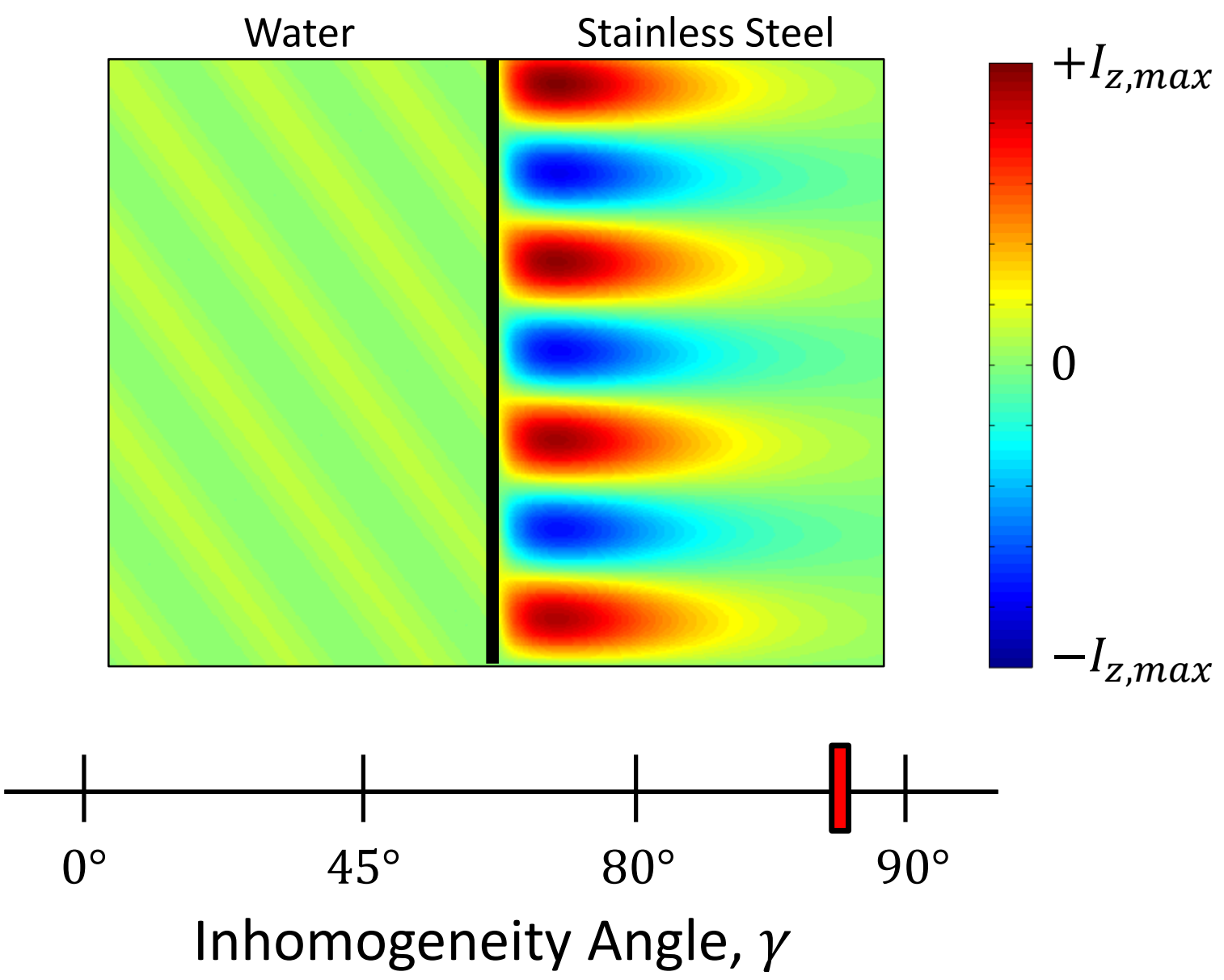


\section{High-Loss Interface: Air-Sylgard}

$|\tilde{R}|$

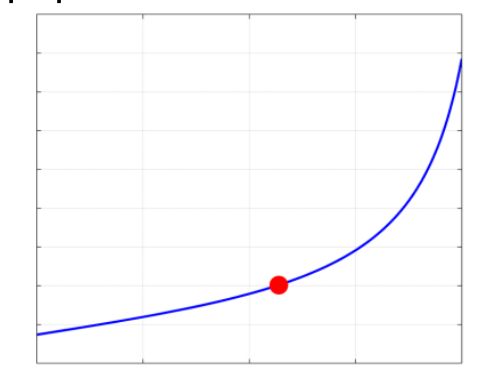

Inhomogeneity Angle, $\gamma$

\section{Transmitted Normal Stress Distribution}
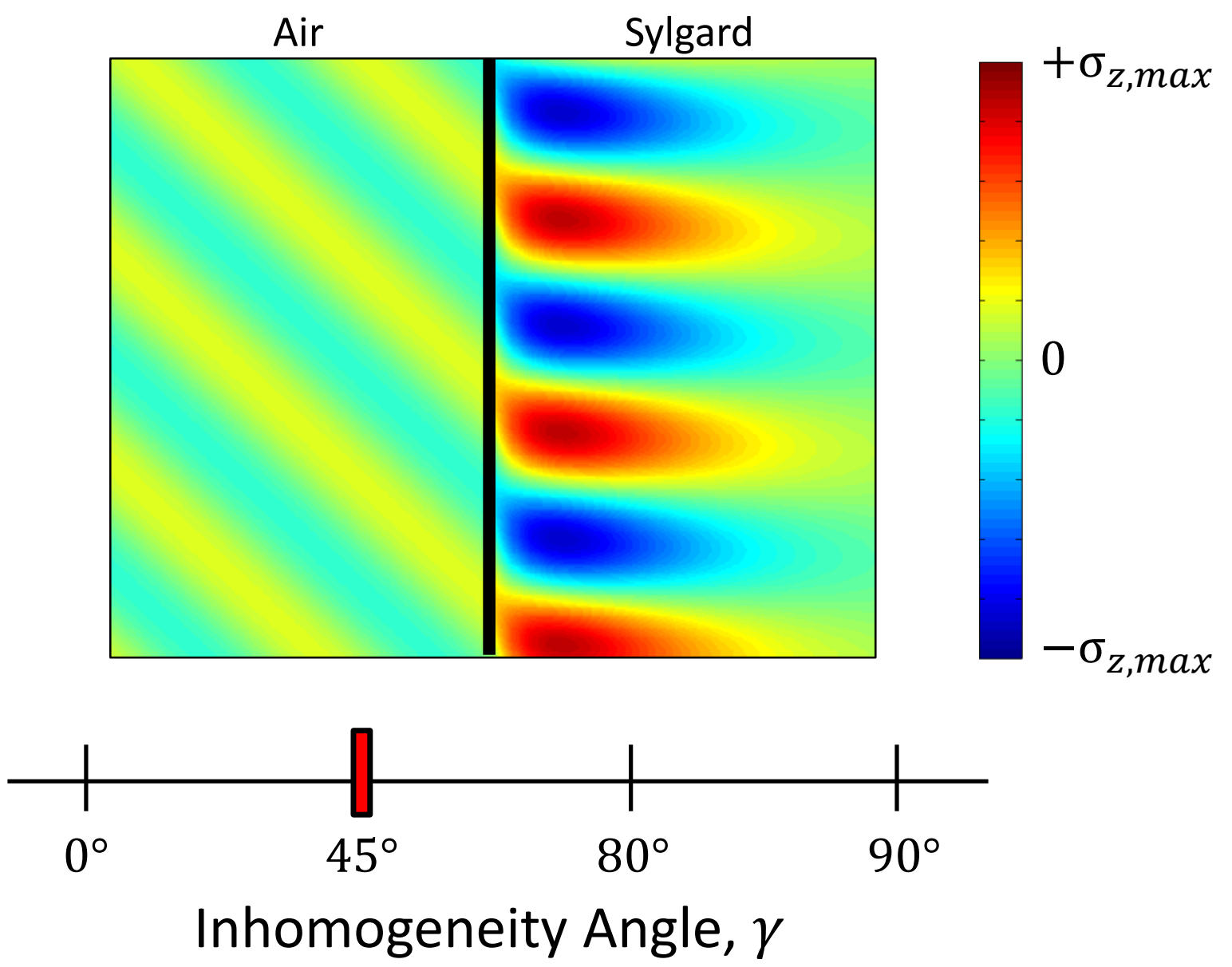


\section{High-Loss Interface: Air-Sylgard}

$|\tilde{R}|$

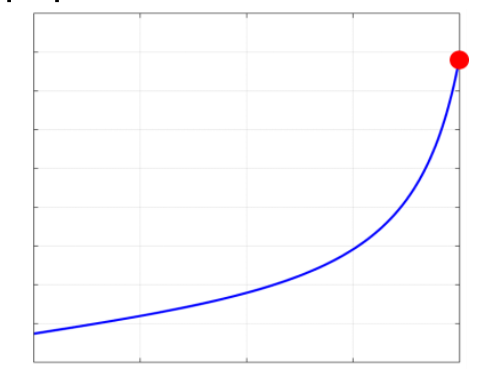

Inhomogeneity Angle, $\gamma$

\section{Transmitted Normal Stress Distribution}
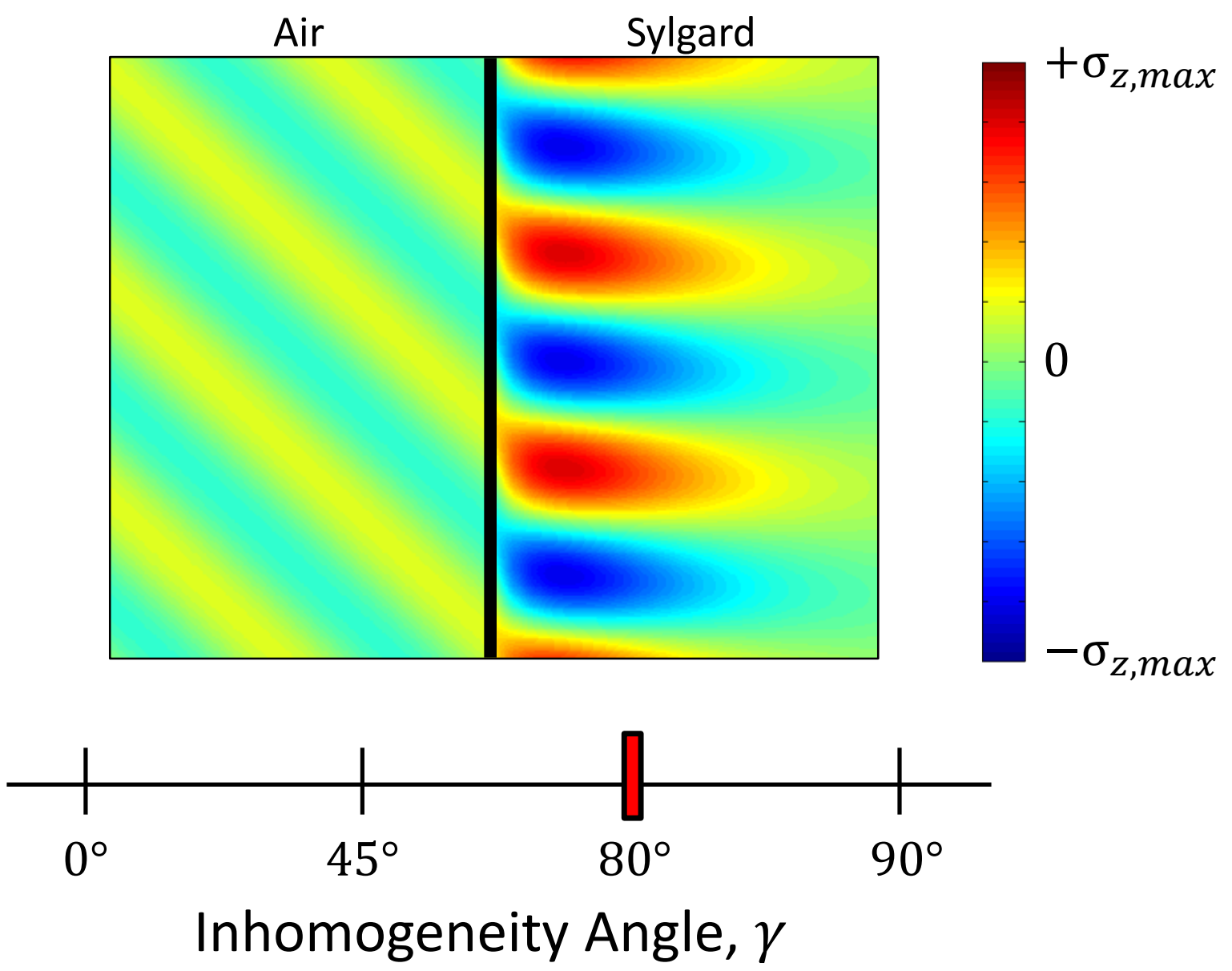


\section{Conclusions}

- Use of general acoustic plane waves for increased transmission in solids

\begin{tabular}{c|c|c} 
Interface & $\begin{array}{c}\text { Optimal incident } \\
\text { wave }\end{array}$ & $\begin{array}{c}\text { Attainable energy } \\
\text { transmission }\end{array}$ \\
\hline $\begin{array}{l}\text { Ideal fluid- } \\
\text { Elastic solid }\end{array}$ & $\begin{array}{l}\text { - Inhomogeneous } \\
\text { At Rayleigh angle }\end{array}$ & $\begin{array}{l}\text { - Total transmission } \\
\text { - Reflection } \rightarrow 0\end{array}$ \\
\hline $\begin{array}{c}\text { Real fluid- } \\
\text { Viscoelastic solid }\end{array}$ & $\begin{array}{l}\text { Inhomogeneous } \\
\text { for low-loss solids } \\
\text { Homogeneous for } \\
\text { high-loss solids }\end{array}$ & $\begin{array}{l}\text { - Less than total } \\
\text { transmission }\end{array}$ \\
& $\begin{array}{l}\text { Reflection }>0 \\
\text { Wider domain }\end{array}$
\end{tabular}




\section{Next Steps}

- Further characterization of transmission into viscoelastic materials of interest

- Polymer-bonded energetic materials

- Transmission by finite, spatially-distributed waves

- Bounded wave profiles typically used in practice

- Various spatial distributions

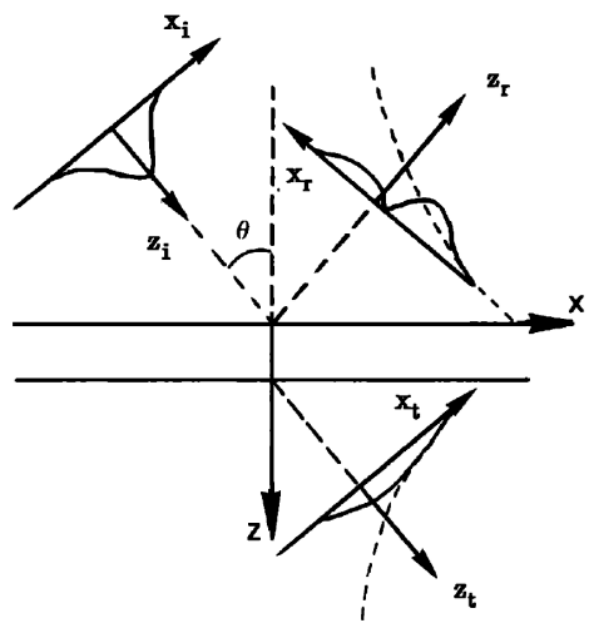




\section{Acknowledgement}

The authors would like to thank the U.S. Office of Naval Research for its support of this research under ONR Grant No. N00014-10-1-0958 


\section{Results for Ideal Fluid-Solid Interface}

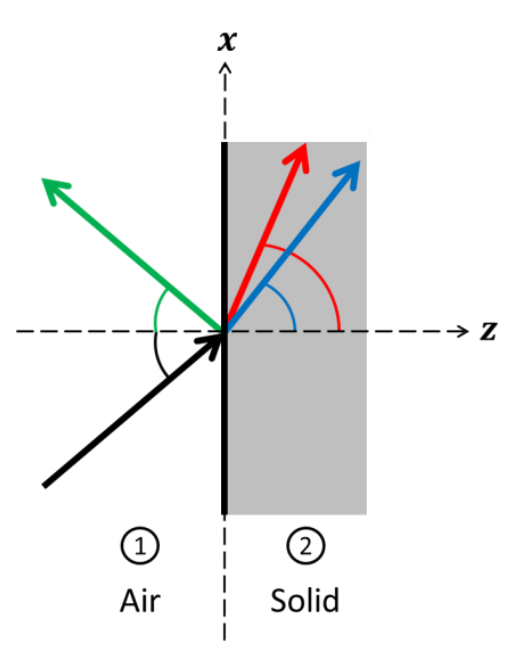

\section{Transmitted Normal Intensity $\left(\mathrm{W} / \mathrm{m}^{2}\right)$}

$1-\mathrm{Pa}, 1000-\mathrm{Hz}$ incident wave

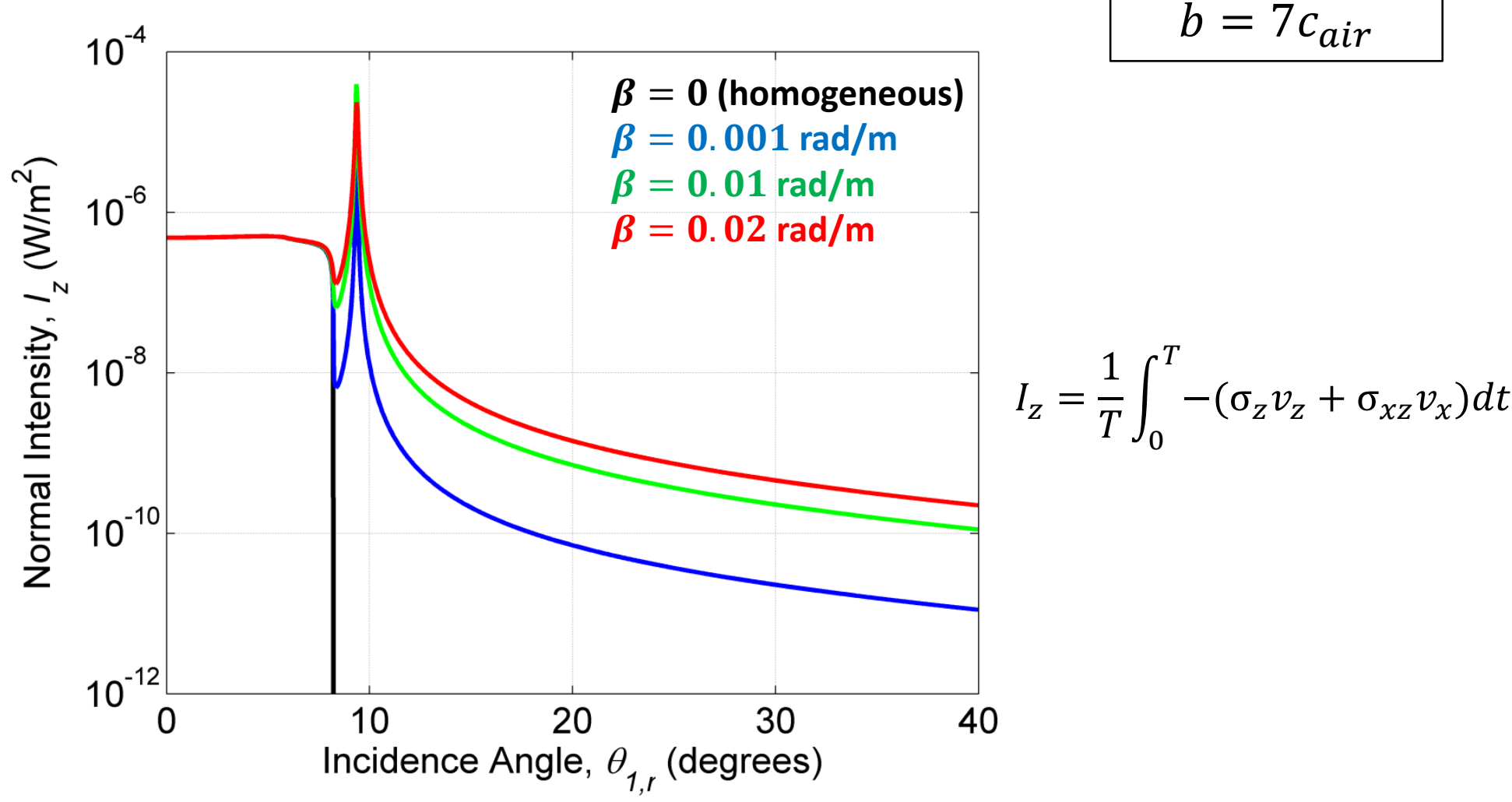




\section{Results for Ideal Fluid-Solid Interface}

Transmitted Normal Stress (Pa)

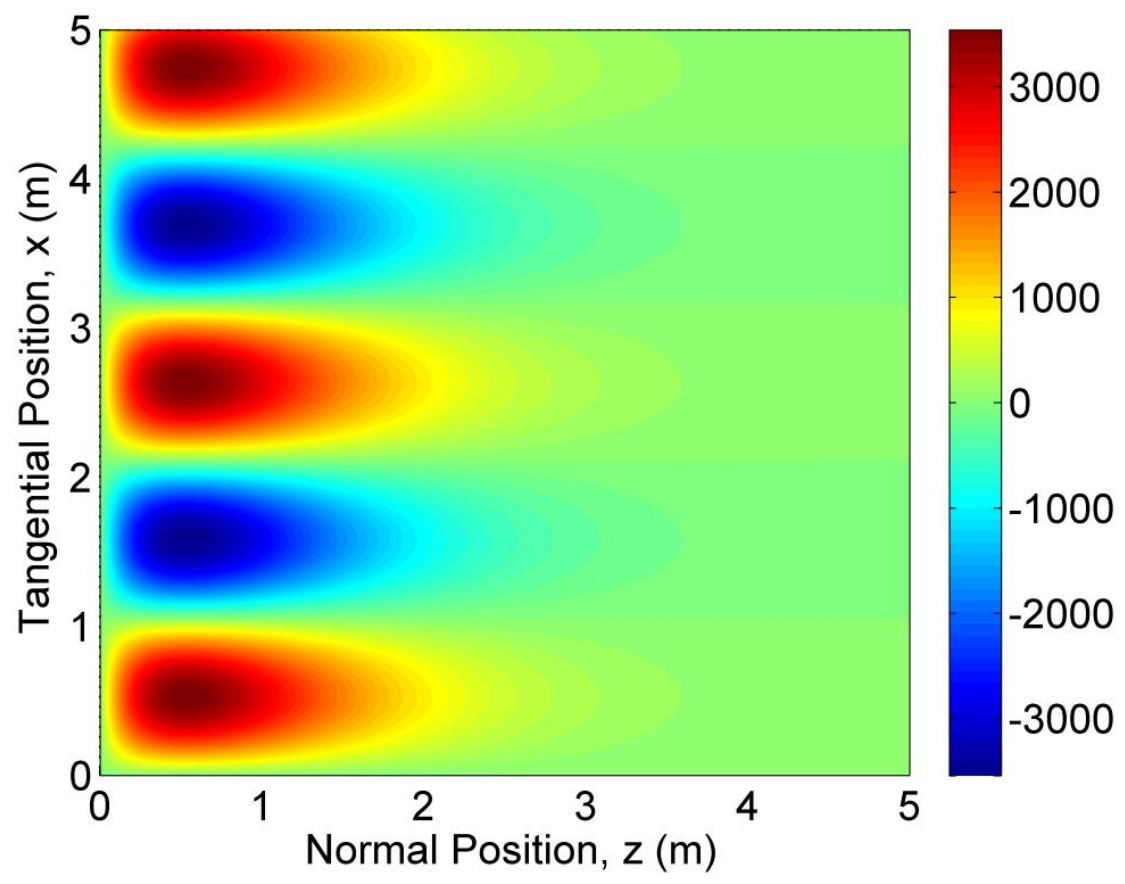

Transmitted Normal Velocity $(\mathrm{m} / \mathrm{s})$

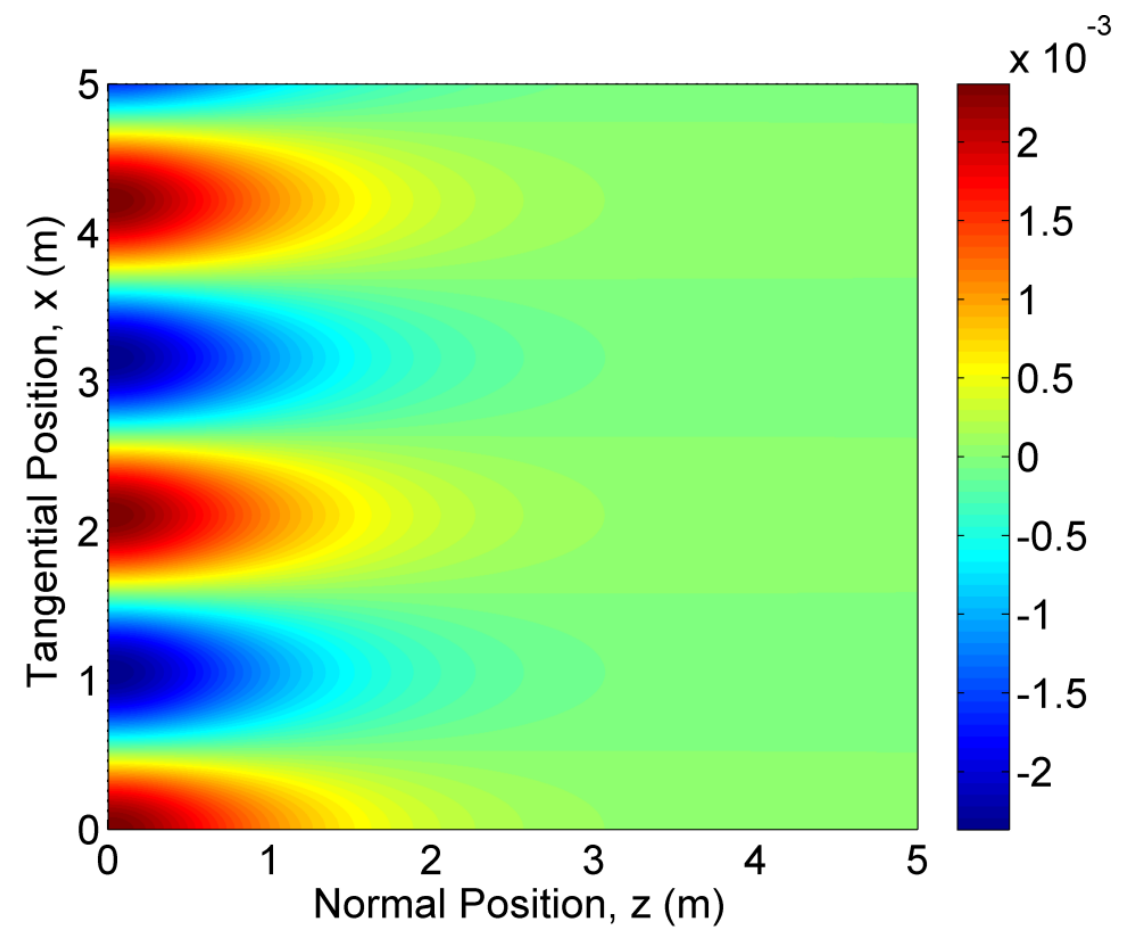

Approximate parameters for $|\widetilde{\boldsymbol{R}}|=\mathbf{0}$

$$
\theta_{1, r}^{*} \approx 9.3657^{\circ}, \beta^{*} \approx 1.07 \times 10^{-4} \mathrm{rad} / \mathrm{m}
$$




\section{Results for Ideal Fluid-Solid Interface}

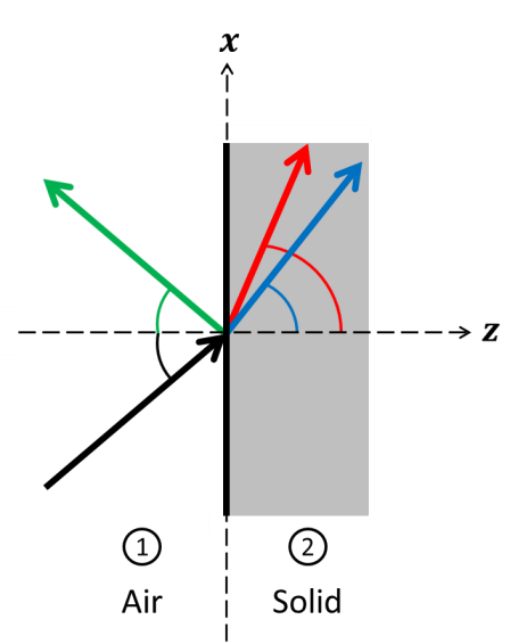

Transmitted Normal Intensity $\left(\mathrm{W} / \mathrm{m}^{2}\right)$

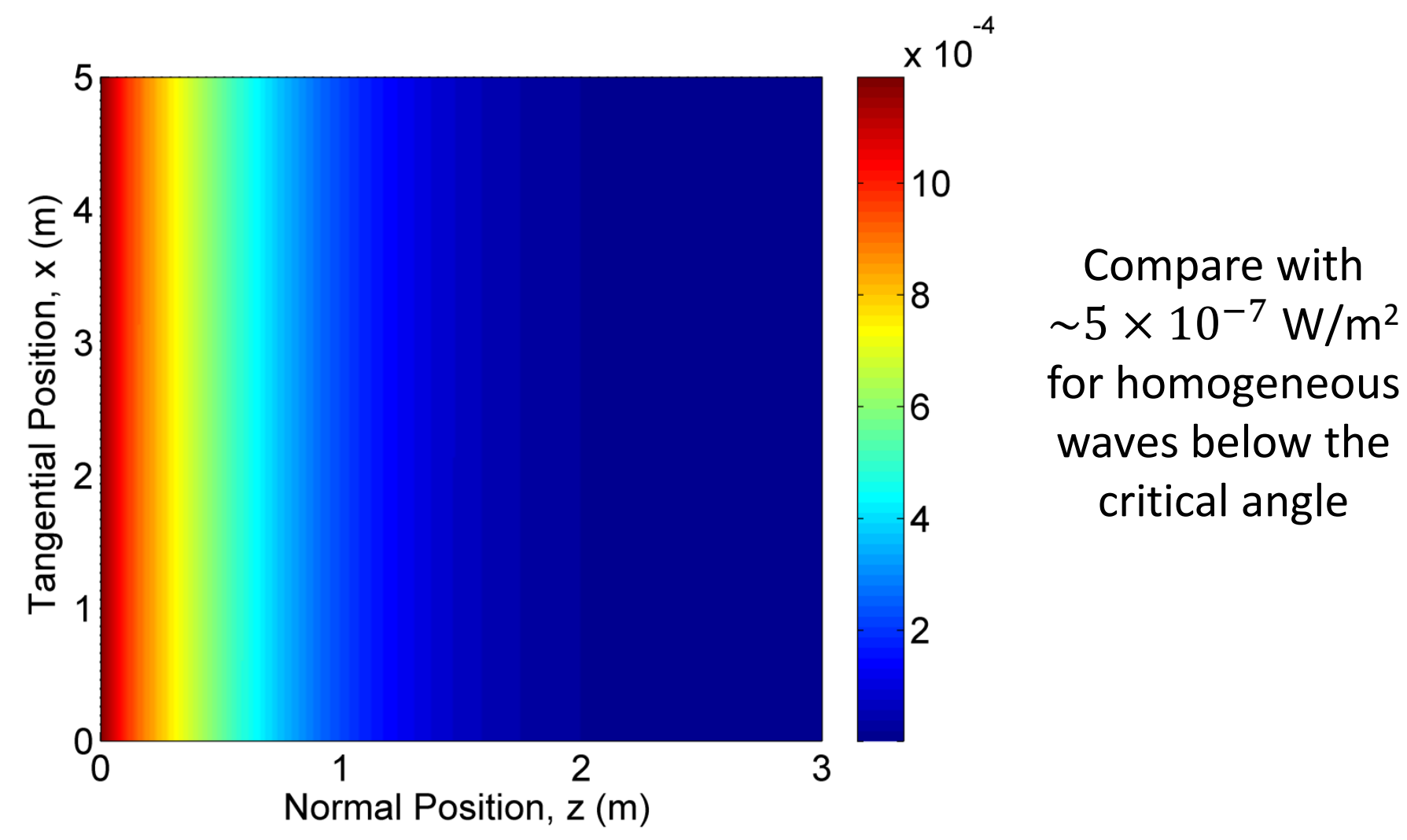

